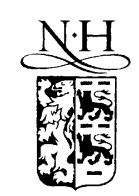

ELSEVIER
Computer methods in applied mechanics and engineering

\title{
Numerical simulation of delamination growth in curved interfaces
}

\author{
Arnaud Münch ${ }^{\mathrm{a}, \mathrm{b}, *}$, Yves Ousset ${ }^{\mathrm{a}}$ \\ a Office National d'Etudes et de Recherches Aérospatiale, BP 72, 92322 Châtillon Cedex, France \\ ${ }^{\mathrm{b}}$ Laboratoire de Modélisation Mécanique, Université Pierre et Marie Curie, 8 rue du capitaine Scott, 75015 Paris Cedex, France
}

Received 18 June 2001

\begin{abstract}
This paper describes a numerical method to simulate delamination growth in layered composite structures within the framework of fracture mechanics in large displacement. It is based on the search for the stationary points of the total energy of the structure, defined as the sum of the mechanical energy and the fracture energy associated with the delamination growth. The resulting non-linear problem is solved by Newton's method. The expressions for the first and second derivatives of energy with respect to a crack front displacement are derived analytically. Numerical examples are presented for specimens loaded in mode I and show the influence of the geometrical parameters on the growth. () 2002 Elsevier Science B.V. All rights reserved.
\end{abstract}

Keywords: Delamination; Fracture mechanics; Numerical analysis

\section{Introduction}

Composites laminates are widely used in both civil and military aircraft structures leading to weight saving. However, study of the behaviour of such materials has shown that they are more damage sensitive than metallic material especially to delamination due to edge effect or low velocity impact [1]. In order to improve the performance of composite structures, advances must be made in the prediction of delamination growth and the evaluation of residual strength. The aim of this paper is to extend a delamination model valid for the plate in small displacement [2] or large displacement [3] to the case of curved structures as shells.

Two kinds of approach are commonly used to study delamination growth, (i) the damage mechanics approach in which the interface enclosing the delamination is modelled by a damageable material. Delamination is obtained when the damage variable reaches its maximum value [4-7] and (ii) the fracture mechanics approach which the present work is part. In such an approach, the delamination characteristic are the stress intensity factors [8-10] and more generally the local energy release rate computed using either

\footnotetext{
${ }^{*}$ Corresponding author. Fax: +33-1-4673-4891.

E-mail addresses: arnaud.munch@onera.fr (A. Münch), yo@onera.fr (Y. Ousset).
} 
the virtual crack closure technique [11-13] or the so-called $\theta$-method proposed by Destuynder [14] and recently extended to a boundary integral equation framework by Bonnet [15]. The $\theta$-method allows the expressions of the energy derivatives with respect to the front displacement to be derived analytically.

To our knowledge, few numerical studies are devoted to delamination growth simulation, within the framework of fracture mechanics. Most of them use of local Griffith's criterion [16]: the local energy release rate $G$ is compared to the critical value $G_{\mathrm{c}}$ and the points of the front where $G>G_{\mathrm{c}}$ are moved along the normal to the front. The incremental law is either empirical and the iterative process is stopped when $G \leqslant G_{\mathrm{c}}$ everywhere along the front [17,18], or derived from the theory of thermodynamics of continuum media [19]. As explained in [2], this local approach must be given up in complex situations for which a global method similar to those of [20-22] is more convenient. Such methods are based on the minimization of the total energy $E$ defined as the sum of the mechanical energy and a fracture energy associated to the delamination growth. For a fixed level of loading, if the delamination growth is stable, it is assumed that the front location at arrest is a stationary point for $E$. The resulting non-linear problem is then solved using Newton's method.

In the case of a shell structure the situation is more complex because the delamination front is not moving in a plane but in a curved surface $\omega$ of the Euclidean space [23]. For this reason, in order to derive the mechanical energy in respect to the evolution of the crack front, we need to express first the formulation in a local reference frame associated to $\omega$ [24]. It is made in Section 2. The expressions of the energy derivatives are then derived in Section 3. This allows to apply in Section 4 the delamination growth model previously developed in the case of plane delaminations. The numerical approximation is presented in Section 5. Finally, numerical examples are studied in Section 6 and the influence of the geometrical parameters on the growth is highlighted. To our knowledge, beyond the few works concerning delamination in curved structure, no one has been studied this influence. Let us mention (i) the experimental works of Ozdil Carlsson [25] and Davies et al. [26] who determined the critical energy release rate associated to a crack in composite cylindrical structures; (ii) the theoretic works of Storackers and Larsson [27] who studied the behaviour of a cylindrical structure with a circular delamination and the works of Rankin et al. [28] who studied the behaviour of cracks in stiffened fuselage shells.

\section{Problem statement}

Let us consider, in the Euclidean space $\mathbb{E}^{3}$ referred to the orthonormal frame $\left(O ; \vec{e}_{1}, \vec{e}_{2}, \vec{e}_{3}\right)$ a cantilever layered shell $\Omega$ of constant thickness $2 h$ having a delamination at an interface $\omega$ parallel to its mid-surface (see Fig. 1 for notations). The structure is fixed on the part $\Gamma_{u} \subset \partial \Omega$ where $\partial \Omega$ is the boundary of $\Omega$ and is submitted to the surface loads $f \in\left(L^{2}\left(\Gamma_{f}\right)\right)^{3}$ on $\Gamma_{f} \subset \partial \Omega$ such that $\Gamma_{u} \cap \Gamma_{f}=\emptyset$. It is assumed that (i) the neighbourhood of the delamination front $\gamma_{f}$ is unloaded, (ii) the loading is proportional i.e. $f=\lambda t$ where $\lambda$ is the loading factor and $t$ is a fixed loading, (iii) the total energy $E$ of the shell is the sum of the mechanical energy $J$ and a fracture energy $D$ associated with the delamination propagation

$$
E\left(u, \gamma_{f}\right)=J\left(u, \gamma_{f}\right)+D\left(u, \gamma_{f}\right)
$$

The functionals $J$ and $D$ depend generally on both the displacement field $u$ and the front location $\gamma_{f}$. Nevertheless, in order to concentrate our study essentially on the curvilinear aspect of the structure and of the delamination, we will assume that the functional $D$ is independent of the displacement field $u$. On this subject, we refer the reader to the recent work of [29].

The model of delamination growth is stated as follows: let the delamination front $\gamma_{f}^{0} \subset \omega$ at starting and the loading magnitude $f$ be known and unchanged during propagation; it is assumed that the unknown front at arrest $\gamma_{f}$ and the displacement field $u$ ensure the stationarity of $E(u, \gamma)$ seen as a functional of two variables; 


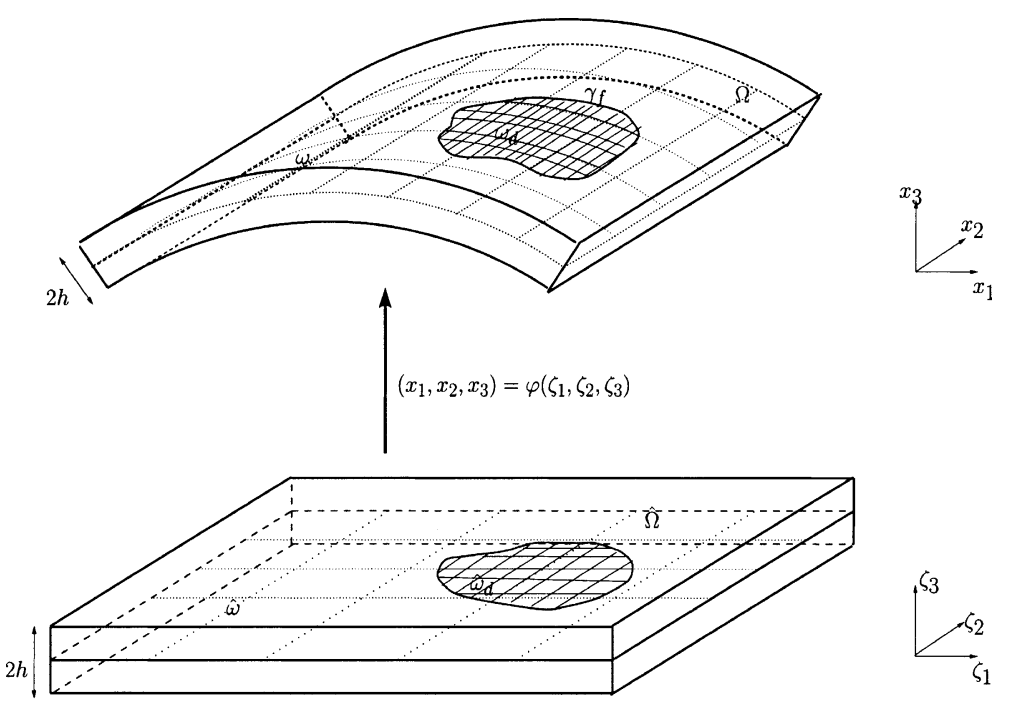

Fig. 1. Representation of the delaminated structure in the global $\left(x_{1}, x_{2}, x_{3}\right)$ and local $\left(\zeta_{1}, \zeta_{2}, \zeta_{3}\right)$ reference frames.

$$
\left(u, \gamma_{f}\right) ? \quad \text { such that } E\left(u, \gamma_{f}\right)=\min _{(v, \gamma)} E(v, \gamma) \quad \forall v \in V, \forall \gamma \in S
$$

where $V$ and $S$ will be specified later.

\subsection{The elasticity problem: global reference frame}

\subsubsection{Mechanical energy $J\left(u, \gamma_{f}\right)$}

A Lagrangian formulation is used to describe the large displacements motion of the structure and it is assumed that the elastic materials are of the St. Venant-Kirchhoff type. The constitutive equations are then linear relations between the Green-Lagrange strain tensor $\gamma(u)$ and the second Piola-Kirchhoff stress tensor $\sigma$

$$
\sigma=R: \gamma(u), \quad \gamma(u)=\frac{1}{2}\left(F^{\mathrm{t}} \cdot F-I_{3}\right), \quad F=I_{3}+\nabla u,
$$

where $R$ is the fourth order material stiffness tensor and let $S$ be its inverse; $F$, the gradient of the transformation $x \rightarrow x+u(x) ; I_{3}$, the identity tensor of $\mathbb{E}^{3}$ and $\nabla u=\left(\partial u_{i} / \partial x_{j}\right), i, j=1, \ldots, 3$. Then, the mechanical energy of the structure is written as

$$
J\left(u, \gamma_{f}\right)=\frac{1}{2} \int_{\Omega\left(\gamma_{f}\right)} \operatorname{Tr}(\sigma \cdot \gamma(u)) \mathrm{d} \Omega-\lambda \int_{\Gamma_{f}} t \cdot u \mathrm{~d} \Gamma
$$

where $\operatorname{Tr}$ is the trace operator. The minimum of $J$, and $E$, with respect to $u$ is then characterized by the following variational problem

$$
\begin{cases}\int_{\Omega} \operatorname{Tr}(S: \sigma \cdot \tau) \mathrm{d} \Omega=\int_{\Omega} \operatorname{Tr}(\tau \cdot \gamma(u)) \mathrm{d} \Omega & \forall \tau \in \Sigma(\Omega), \\ \int_{\Omega} \operatorname{Tr}\left(\sigma \cdot F^{\mathrm{t}} \cdot \partial v / \partial x\right) \mathrm{d} \Omega=\lambda \int_{\Gamma_{f}} t \cdot v \mathrm{~d} \Gamma & \forall v \in V(\Omega),\end{cases}
$$

with $V(\Omega)=\left\{v \in\left(W^{1,4}(\Omega)\right)^{3} / v_{\mid \Gamma u}=0\right\}$ and $\Sigma(\Omega)=\left(L_{s}^{2}(\Omega)\right)^{9}$ [30]. We assume that the formulation (5) has at least one solution $(u, \sigma)$. In order to prevent interpenetration, the space $V(\Omega)$ of the admissible displacement fields should be reduced to 


$$
V_{\mathrm{g}}(\Omega)=\left\{v \in\left(W^{1,4}(\Omega)\right)^{3} / v_{\mid \Gamma_{u}}=0 / g(v) \geqslant 0 \text { on } \omega_{\mathrm{d}} \subset \omega\right\}
$$

where the function $g(u)$ describes the displacement gap at any point of the delaminated part $\omega_{\mathrm{d}} \subset \omega$.

\subsubsection{Fracture energy $D\left(\gamma_{f}\right)$}

According to the Griffith's assumption, it is assumed that the fracture energy associated with delamination propagation is proportional to the increment of the delaminated area. If ${ }^{\mathrm{c}} \omega_{\mathrm{d}}^{1}=\omega^{1}-\omega_{\mathrm{d}}^{1}$ is the undelaminated part of the interface at starting and ${ }^{\mathrm{c}} \omega_{\mathrm{d}}$ the current part, the fracture energy is defined as

$$
D\left(\gamma_{f}\right)=G_{\mathrm{c}}\left(\int_{\mathrm{c}_{\omega_{\mathrm{d}}^{1}}^{1}} \mathrm{~d} \omega-\int_{\mathrm{c}_{\omega_{\mathrm{d}}}} \mathrm{d} \omega\right) .
$$

$G_{\mathrm{c}}$ is the critical energy release rate assumed constant along $\gamma_{f}$.

\subsection{The elasticity problem: local reference frame}

In order to derive easily the total energy with respect to the crack front displacement, we express the problem in a local reference frame associated to the delaminated interface $\omega$. In the following, the repeated summation convention is used: latin $i, j, k, \ldots$ (resp. greek $\alpha, \beta, \ldots$ ) indices and exponents take their values in the set $\{1,2,3\}$ (resp. $\{1,2\}$ ).

\subsubsection{Local description of the structure}

Let us assume that the delaminated interface is defined by an injective mapping $\phi: \overline{\hat{\omega}} \in \mathbb{R}^{2} \rightarrow \mathbb{E}^{3}$ such that:

$$
\forall x=\left(x_{1}, x_{2}, x_{3}\right) \in \omega, \quad \exists \zeta_{\alpha}=\left(\zeta_{1}, \zeta_{2}\right) \in \hat{\omega} ; \quad x=\phi\left(\zeta_{\alpha}\right),
$$

the tangent space at $x$ to $\omega$ is then defined by the vectors (assumed linearly independents) $\vec{a}_{\alpha}=\partial_{\alpha} \phi$. The unit normal $\vec{a}_{3}$ to $\omega$ at $x$ is then defined as $\vec{a}_{3}=\left(\vec{a}_{1} \wedge \vec{a}_{2}\right) /\left(\left\|\vec{a}_{1} \wedge \vec{a}_{2}\right\|\right)(\wedge$ is the exterior product). The vectors $\left(\vec{a}_{\alpha}, \vec{a}_{3}\right)$ form the covariant basis at $x$. Let $\hat{\Omega}$ be the open set defined as

$$
\hat{\Omega}=\hat{\omega} \times]-h^{\mathrm{I}}, h^{\mathrm{S}}[,
$$

where $h^{\mathrm{I}}$ and $h^{\mathrm{S}}$ are the thickness of the lower and upper delaminated parts of $\Omega$ respectively $\left(h^{\mathrm{I}}+h^{\mathrm{S}}=2 h\right)$, and let be $\zeta=\left(\zeta_{\alpha}, \zeta_{3}\right) \in \hat{\Omega}$, then the domain $\Omega$ admits the following parametric description at $x$ :

$$
\Omega=\left\{x \in \mathbb{E}^{3} / x=\phi\left(\zeta_{\alpha}\right)+\zeta_{3} \vec{a}_{3} ; \zeta \in \hat{\Omega}\right\} .
$$

We note $a_{\alpha \beta}=\vec{a}_{\alpha} \vec{a}_{\beta}$ the covariant components of the metric tensor, $b_{\alpha \beta}=\vec{a}_{3} \vec{a}_{\alpha, \beta}$ the covariant components of the curvature tensor. The associated contravariant basis $\left(\vec{a}^{\alpha}, \vec{a}^{3}\right)$ is then defined as $a^{\alpha}=a^{\alpha \beta} a_{\beta}$ and $\vec{a}^{3}=\vec{a}_{3}^{\mathrm{t}}$. We choose to express the displacement field $\vec{u}$ in the contravariant bases

$$
\vec{u}(x)=u^{i}(x) \vec{e}_{i}=u_{i}(\zeta) \vec{a}^{i}(\zeta)=\overrightarrow{\hat{u}}(\zeta),
$$

and the two times contravariant stress tensor $\sigma$ such that

$$
\sigma(x)=\sigma_{i j}(x) \vec{e}_{i} \otimes \vec{e}_{j}=\sigma^{i j}(\zeta) \vec{a}_{i}(\zeta) \otimes \vec{a}_{j}(\zeta)=\hat{\sigma}(\zeta) .
$$

We refer the reader to [30] for the complete description.

\subsubsection{Mechanical energy $\hat{J}\left(\underline{u}, \hat{\gamma}_{f}\right)$ and fracture energy $\hat{D}\left(\hat{\gamma}_{f}\right)$}

After a straightforward computation, the variational formulation (5) takes the following form (see Appendix A for details and notations): 


$$
\begin{cases}\int_{\hat{\Omega}} \operatorname{Tr}(\underline{S}: \underline{\sigma} \cdot \underline{\tau})=\int_{\hat{\Omega}} \operatorname{Tr}(\underline{\tau} \cdot \underline{\gamma}(\underline{u})) & \forall \underline{\tau} \in \Sigma(\hat{\Omega}), \\ \int_{\hat{\Omega}} \operatorname{Tr}\left(\underline{\sigma} \cdot \underline{F} \cdot(\underline{d} \cdot e(\hat{v}))^{\mathrm{t}}\right)=\lambda \int_{\hat{\Gamma}_{f}} \hat{t} \cdot \hat{v} \sqrt{|g|} \mathrm{d} \Gamma & \forall \hat{v} \in V(\hat{\Omega}),\end{cases}
$$

where the components of the tensor $\underline{S}$ are generally functions of the coordinates $\zeta$, even in the case of isotropic materials. In this formulation, the delamination front $\gamma_{f}$ has a planar representation $\hat{\gamma}_{f} \in \mathbb{R}^{2}$ such that $\phi\left(\hat{\gamma}_{f}\right)=\gamma_{f}$ which permits us to apply the method used in the plane case [2,3]. The associated energy is expressed as

$$
\hat{J}\left(\underline{u}, \hat{\gamma}_{f}\right)=\frac{1}{2} \int_{\hat{\Omega}\left(\gamma_{f}\right)} \operatorname{Tr}(\underline{\sigma} \cdot \underline{\gamma}(u)) \mathrm{d} \hat{\Omega}-\lambda \int_{\hat{\Gamma}_{f}} \hat{t} \cdot \hat{u} \sqrt{|g|} \mathrm{d} \hat{\Gamma},
$$

whereas the fracture energy (7) becomes

$$
\hat{D}\left(\hat{\gamma}_{f}\right)=G_{\mathrm{c}}\left(\int_{\mathrm{c}_{\hat{\omega}_{\mathrm{d}}^{1}}} \sqrt{|g|} \mathrm{d} \hat{\omega}-\int_{\mathrm{c}_{\hat{\omega}_{\mathrm{d}}}} \sqrt{|g|} \mathrm{d} \hat{\omega}\right) .
$$

\section{Energies derivatives}

In the following, the tensors are functions of the local coordinates $\zeta$ and we note $u$ for $\underline{u}$ and $\sigma$ for $\underline{\sigma}$ the displacement field and the strain tensor.

\subsection{The $\theta$-method}

We now have to write the stationarity of $E$ with respect to $\hat{\gamma}_{f}$. As it is assumed that the delamination front $\hat{\gamma}_{f}$ remains at the interface $\hat{\omega} \subset \mathbb{R}^{2}$ during propagation, the virtual kinematics of the delamination front can be described by a plane vector field $\theta(\zeta)=\left(\theta_{1}(\zeta), \theta_{2}(\zeta), 0\right)$ where the covariant components $\theta_{\alpha}$ are regular functions defined in $\hat{\Omega}$ with support $S_{\theta}$ restricted to a small neighbourhood of $\hat{\gamma}_{f}$ such that $S_{\theta} \cap \hat{\Gamma}_{f}=\emptyset$. If the front intersects the boundary of $\hat{\omega}$ (see Fig. 2), $\theta$ must satisfy the kinematical condition

$$
\theta \cdot v=0 \text { on } S_{\theta} \cap \partial \hat{\omega},
$$

where $v$ is the unit normal. Let $\eta$ be a dimensionless small parameter. It is associated to the field $\theta$ a family of mappings

$$
\mathscr{F}^{\eta}: \zeta \in \hat{\Omega} \rightarrow \zeta^{\eta} \in \hat{\Omega}^{\eta} ; \quad \zeta^{\eta}=\zeta+\eta \theta(\zeta)
$$

Let $(u, \sigma)$ and $\left(u^{\eta}, \sigma^{\eta}\right)$ be the solution of problem (13) set in $\hat{\Omega}$ and $\hat{\Omega}^{\eta}$ respectively for the same loading factor $\lambda$. The first and second derivatives of the energy $E$ with respect to the front displacement in the $\theta$ direction are defined as
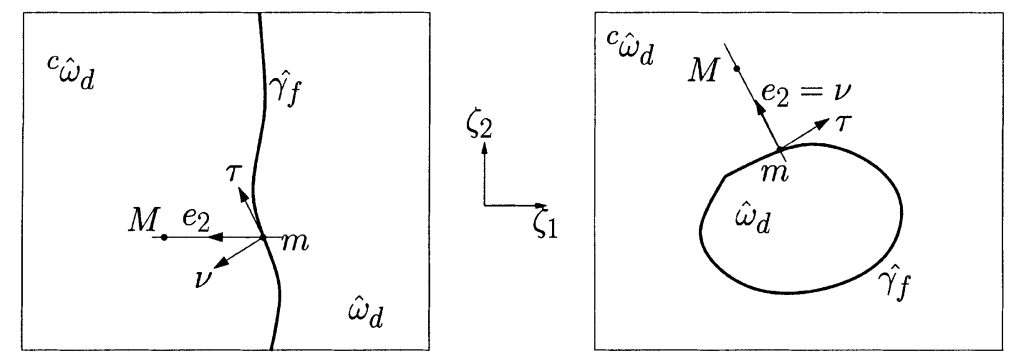

Fig. 2. Definition of the projection: front crossing over the specimen width $\left(\hat{\gamma}_{f} \cap \partial \hat{\omega} \neq \emptyset\right)$ and internal delamination $\left(\hat{\gamma}_{f} \cap \partial \hat{\omega}=\emptyset\right)$. 


$$
E^{(1)}(\theta)=\lim _{\eta \rightarrow 0} \frac{E^{\eta}-E}{\eta} ; \quad E^{(2)}(\theta, \theta)=\lim _{\eta \rightarrow 0} \frac{E^{\eta}-E-\eta E^{(1)} \cdot \theta}{\eta^{2}},
$$

and are obtained in a classical way [14,31]: first, the integrals and derivatives over $\hat{\Omega}^{\eta}$ are expressed as integrals and derivatives over $\hat{\Omega}$ using the two following relations:

$$
\begin{aligned}
\mathrm{d} \zeta^{\eta} & =\operatorname{det}\left(I_{2}+\eta \nabla \theta\right) \mathrm{d} \zeta=\left(1+\eta \operatorname{div} \theta+\eta^{2} \operatorname{det}(\nabla \theta)\right) \mathrm{d} \zeta, \\
\frac{\partial}{\partial \zeta^{\eta}} & =\frac{\partial}{\partial \zeta}\left(I_{2}+\eta \nabla \theta\right)^{-1}=\frac{\partial}{\partial \zeta}\left(I_{2}-\eta \nabla \theta+\eta^{2} \nabla \theta \cdot \nabla \theta\right)+\mathrm{o}\left(\eta^{2}\right),
\end{aligned}
$$

making clear the $\eta$ dependence. Then the solution $\left(u^{\eta}, \sigma^{\eta}\right)$ is expanded as a formal power series of $\eta$ :

$$
\left(u^{\eta}, \sigma^{\eta}\right)=(u, \sigma)+\eta\left(u^{1}, \sigma^{1}\right)+\eta^{2}\left(u^{2}, \sigma^{2}\right)+\mathrm{o}\left(\eta^{2}\right),
$$

and terms of same order are identified. In the curvilinear case [24], we have to develop the local metric induced by the map $\phi$. For example, for the covariant metric tensor, we write $a^{\eta}=a+\eta \nabla a \cdot \theta+$ $\eta^{2} \frac{1}{2} \theta \cdot \nabla^{2} a \cdot \theta+\mathrm{o}\left(\eta^{2}\right)$, this relation having to be considered component by component. We give now the results of the computation.

\subsection{First order Lagrangian derivatives}

The first order Lagrangian derivatives $\left(u^{1}, \sigma^{1}\right)$ are needed to compute the second derivative of $J$; they are the solution of the following variational problem $\forall v \in V(\hat{\Omega})$

$$
\left\{\begin{aligned}
& \sigma^{1}= \underline{R}: \underline{F} \cdot\left(\left[d \cdot e\left(u^{1}\right)\right]^{\mathrm{t}}+\left[d \cdot\left(\nabla f(u) \cdot \theta-(\nabla u \cdot \nabla \theta)^{\mathrm{t}}\right)\right]^{\mathrm{t}}+[\nabla d \cdot \theta \cdot e(u)]^{\mathrm{t}}\right) \\
&-\underline{R}: \nabla \underline{S} \cdot \theta \cdot \underline{R}: \gamma(u)+\frac{1}{2} R: d \cdot e(u) \cdot \nabla a \cdot \theta \cdot[d \cdot e(u)]^{\mathrm{t}}, \\
& \int_{\hat{\Omega}} \operatorname{Tr}\left(\sigma^{1} \cdot \underline{F} \cdot[d \cdot e(v)]^{\mathrm{t}}\right)+\operatorname{Tr}\left(\sigma \cdot d \cdot e\left(u^{1}\right) \cdot a \cdot[d \cdot e(v)]^{\mathrm{t}}\right) \\
&=-\int_{\hat{\Omega}} \operatorname{Tr}\left(\sigma \cdot \underline{F} \cdot[d \cdot e(v)]^{\mathrm{t}}\right) \operatorname{div} \theta-\int_{\hat{\Omega}} \operatorname{Tr}\left(\sigma \cdot \underline{F} \cdot[\nabla d \cdot \theta \cdot e(v)]^{\mathrm{t}}\right) \\
& \quad-\int_{\hat{\Omega}} \operatorname{Tr}\left(\sigma \cdot \underline{F} \cdot\left[d \cdot\left(\nabla f(v) \cdot \theta-(\nabla v \cdot \nabla \theta)^{\mathrm{t}}\right)\right]^{\mathrm{t}}\right)-\int_{\hat{\Omega}} \operatorname{Tr}\left(\sigma \cdot d \cdot e(u) \cdot \nabla a \cdot \theta \cdot[d \cdot e(v)]^{\mathrm{t}}\right) \\
& \quad-\int_{\hat{\Omega}} \operatorname{Tr}\left(\sigma \cdot \nabla d \cdot \theta \cdot e(u) \cdot a \cdot[d \cdot e(v)]^{\mathrm{t}}\right)-\int_{\hat{\Omega}} \operatorname{Tr}\left(\sigma \cdot d \cdot\left(\nabla f(u) \cdot \theta-(\nabla u \cdot \nabla \theta)^{\mathrm{t}}\right) \cdot a \cdot[d \cdot e(v)]^{\mathrm{t}}\right),
\end{aligned}\right.
$$

The last equation shows that the Lagrangian derivatives are the solution of a linear problem involving the tangent stiffness operator of problem (13), whereas in the loading term (the right-hand side of the relation), $\theta$ acts as a parameter.

\subsection{Energy release rate}

Let us recall that the energy release rate is defined as $\hat{g}(\theta)=-J^{(1)}(\theta)$. After classical computation, the energy release rate is expressed as a function of the displacement $u$ and the stress $\sigma$ only.

$$
\begin{aligned}
\hat{g}(\theta)= & \frac{1}{2} \int_{\hat{\Omega}} \operatorname{Tr}(\nabla \underline{S}: \theta \cdot \sigma \cdot \sigma)+\int_{\hat{\Omega}} \operatorname{Tr}\left(\sigma \cdot F \cdot\left[d \cdot\left((\nabla u \cdot \nabla \theta)^{\mathrm{t}}-\nabla f(u) \cdot \theta\right)\right]^{\mathrm{t}}\right) \\
& -\frac{1}{2} \int_{\hat{\Omega}} \operatorname{Tr}(\sigma \cdot \underline{\gamma}(u)) \operatorname{div} \theta-\int_{\hat{\Omega}} \operatorname{Tr}\left(\sigma \cdot \underline{F} \cdot[\nabla d \cdot \theta \cdot e(u)]^{\mathrm{t}}\right) \\
& -\frac{1}{2} \int_{\hat{\Omega}} \operatorname{Tr}\left(\sigma \cdot d \cdot e(u) \cdot \nabla a \cdot \theta \cdot[d \cdot e(u)]^{\mathrm{t}}\right) .
\end{aligned}
$$

When the map $\phi$ is equal to the identity, the usual expression in the plane case [3] is recovered. Integrating by parts the terms in $\nabla \theta$ and using both the constitutive and the equilibrium equations, it is found that the energy release rate can be written as a curvilinear integral along the delamination front 


$$
\hat{g}(\theta)=\int_{\hat{\gamma}_{f}} G \sqrt{|g|} \theta \cdot v \mathrm{~d} \Gamma
$$

where $G$ is the so-called delamination force or local energy release rate. For the details of the computation in the 2D case, see [24]. $G$ can be viewed as the dual variable of $\theta \cdot v$ on $\hat{\gamma}_{f}$. This shows that the energy release rate depends on the value of $\theta$ along the delamination front only. For numerical computations, expression (21) is used as it is less mesh sensitive than expression (22). It gives accurate results with relatively coarse finite elements meshes. Lastly, let us note that, due to the presence of $\nabla f$ in $(21)$, a $C^{3}(\hat{\omega})$ regularity is required for the map $\phi$.

\subsection{Mechanical energy second derivative}

The mechanical energy second derivative is obtained identifying the second order terms in $\eta$ in the $J^{\eta}$ expansion. We obtain the following expression, given to simplify, in the case of a constant metric $(\nabla a \equiv 0)$

$$
\begin{aligned}
2 J^{(2)}(\theta, \theta)= & \int_{\hat{\Omega}} \operatorname{Tr}(\sigma \cdot \underline{\gamma}(u)) \operatorname{det}(\nabla \theta)+\int_{\hat{\Omega}} \operatorname{Tr}\left(\sigma^{1} \cdot \underline{\gamma}(u)\right) \operatorname{div} \theta+2 \int_{\hat{\Omega}} \operatorname{Tr}\left(\sigma \cdot \underline{F} \cdot \nabla u(\nabla \theta)^{2} \cdot d^{\mathrm{t}}\right) \\
& -\int_{\hat{\Omega}} \operatorname{Tr}\left(\sigma \cdot \underline{F} \cdot \nabla u^{1} \cdot \nabla \theta d^{\mathrm{t}}\right)-\int_{\hat{\Omega}} \operatorname{Tr}\left(\sigma \cdot \underline{F} \cdot \nabla u \cdot \nabla \theta \cdot d^{\mathrm{t}}\right) \operatorname{div} \theta-\int_{\hat{\Omega}} \operatorname{Tr}\left(\sigma \cdot d \cdot(\nabla u \cdot \nabla \theta)^{\mathrm{t}} \cdot a \cdot\left[d \cdot e\left(u^{1}\right)\right]^{\mathrm{t}}\right) \\
& +\int_{\hat{\Omega}} \operatorname{Tr}\left(\sigma \cdot d \cdot(\nabla u \cdot \nabla \theta)^{\mathrm{t}} \cdot a \cdot \nabla u \cdot \nabla \theta \cdot d^{\mathrm{t}}\right)-\int_{\hat{\Omega}} \operatorname{Tr}\left(\sigma^{1} \cdot \underline{F} \cdot \nabla u \cdot \nabla \theta d^{\mathrm{t}}\right)
\end{aligned}
$$

$J^{(2)}$ is a quadratic form associated to a symmetric bilinear form that depends on the solution $(u, \sigma)$ and on the first Lagrangian derivatives $\left(u^{1}, \sigma^{1}\right)$.

\subsection{Fracture energy derivatives}

In view of the definition of $D$, the derivation of the expansion for the fracture energy derivatives is straightforward. The following expressions are obtained

$$
\left\{\begin{array}{l}
\hat{D}^{(1)}(\theta)=-G_{\mathrm{c}} \int_{\hat{c}_{\hat{\omega}_{\mathrm{d}}}}(\sqrt{|g|} \operatorname{div} \theta+\nabla \sqrt{|g|} \cdot \theta) \mathrm{d} \hat{\omega}, \\
\hat{D}^{(2)}(\theta, \theta)=-G_{\mathrm{c}} \int_{\mathfrak{c}_{\hat{\omega}_{\mathrm{d}}}}\left(\sqrt{|g|} \operatorname{det}(\nabla \theta)+\nabla \sqrt{|g|} \cdot \theta \operatorname{div} \theta+\frac{1}{2} \theta \cdot \nabla^{2} \sqrt{|g|} \cdot \theta\right) \mathrm{d} \hat{\omega} .
\end{array}\right.
$$

According to the identity $\operatorname{div}(\sqrt{|g|} \theta)=\sqrt{|g|} \operatorname{div} \theta+\nabla \sqrt{|g|} \theta$, and using Green's formula, expressions (24) can be put under the form of curvilinear integrals along the front $\hat{\gamma}_{f}$

$$
\left\{\begin{array}{l}
\hat{D}^{(1)}(\theta)=G_{\mathrm{c}} \int_{\hat{\gamma}_{f}} \sqrt{|g|} \theta \cdot v \mathrm{~d} \Gamma \\
\hat{D}^{(2)}(\theta, \theta)=\frac{1}{2} G_{\mathrm{c}} \int_{\hat{\gamma}_{f}}(\operatorname{div}(\sqrt{|g|} \theta) \theta \cdot v-\sqrt{|g|} v \cdot \nabla \theta \cdot \theta) \mathrm{d} \Gamma .
\end{array}\right.
$$

\section{Propagation model}

\subsection{Conditions on $\theta$}

The front $\hat{\gamma}_{f}$ cannot move back; this requirement is satisfied if and only if

$$
\theta \cdot e_{2} \geqslant 0
$$


where $\vec{e}_{2}$ is the unit vector in the transverse direction oriented in the direction of delamination growth. It is taken parallel to the lateral edges of $\hat{\omega}$ in the case of open fronts, or as the unit normal to the front in the case of closed front (see Fig. 2). Due to this irreversibility condition, the problem to be solved is a constrained minimization one. It can be solved using a fixed point algorithm.

On the other hand, the mapping introduced in Section 5.1 must be defined in such a way that the delaminated area increases. This requirement takes the following form:

$$
\begin{aligned}
\int_{\mathrm{c}_{\hat{\omega}_{\mathrm{d}}}} \mathrm{d} \hat{\omega}-\int_{\mathrm{c}_{\hat{\omega}_{\mathrm{d}}^{\eta}}} \mathrm{d} \hat{\omega}^{\eta} & =-\eta \int_{\mathrm{c}_{\hat{\omega}_{\mathrm{d}}}} \operatorname{div}(\sqrt{g} \theta) \mathrm{d} \hat{\omega}-\eta^{2} \int_{\mathfrak{c}_{\hat{\omega}_{\mathrm{d}}}}\left(\sqrt{|g|} \operatorname{det}(\nabla \theta)+\nabla \sqrt{|g|} \cdot \theta \operatorname{div} \theta+\frac{1}{2} \theta \cdot \nabla^{2} \sqrt{|g|} \cdot \theta\right) \mathrm{d} \hat{\omega} \\
& \geqslant 0 \quad \forall \eta>0 .
\end{aligned}
$$

A sufficient condition for $\theta$ is then

$$
\left\{\begin{array}{l}
-\int_{\mathfrak{c}_{\hat{\omega}_{\mathrm{d}}}} \operatorname{div}(\sqrt{|g|} \theta) \mathrm{d} \hat{\omega} \geqslant 0, \\
-\int_{\mathfrak{c}_{\hat{\omega}_{\mathrm{d}}}}\left(\sqrt{|g|} \operatorname{det}(\nabla \theta)+\nabla \sqrt{|g|} \cdot \theta \operatorname{div} \theta+\frac{1}{2} \theta \cdot \nabla^{2} \sqrt{|g|} \cdot \theta\right) \mathrm{d} \hat{\omega} \geqslant 0,
\end{array}\right.
$$

which is equivalent, in the case of constant $G_{\mathrm{c}}$ and according to the relation (24), to

$$
D^{(1)}(\theta) \geqslant 0 ; \quad D^{(2)}(\theta, \theta) \geqslant 0 .
$$

The first condition of (28) is satisfied as soon as condition (26) is satisfied. The set $V_{\theta}$ of admissible displacement fields of the front is so

$$
V_{\theta}=\left\{\theta \in\left(H^{1}\left(S_{\theta}\right)\right)^{2}, \theta \cdot e_{2} \geqslant 0 \text { on } \gamma_{f}, \theta \cdot v=0 \text { on } S_{\theta} \cap \partial \hat{\omega}, D^{(2)}(\theta, \theta) \geqslant 0\right\}
$$

If $(|g|)^{1 / 2}$ is constant (a cylindrical arch for instance), the spectrum of the operator $D^{(2)}$ is real and symmetric with respect to zero (see Appendix B). As a consequence, the sets $\left\{\theta ; D^{(2)}(\theta, \theta)>0\right\}$ and $V_{\theta}$ are not empty.

\subsection{Front displacement}

Let us consider stable delamination growth. According to Nguyen [32], the delamination growth is stable, for a fixed level of loading, if $J^{(2)}(\theta, \theta)>0 \forall \theta \in V_{\theta}$. This condition ensures that, in the vicinity of a stable crack front location at arrest, the total energy $E$ is strictly convex: $E^{(2)}(\theta, \theta)=J^{(2)}(\theta, \theta)+$ $D^{(2)}(\theta, \theta)>0$. As a consequence, the growth arrest corresponds to a local minimum of $E$. According to relations (22) and first term of (25) the stationarity of $E$ with respect to a front increment is written as

$$
E^{(1)}(\theta)=\int_{\hat{\gamma}_{f}}\left(G_{\mathrm{c}}-G\right) \sqrt{|g|} \theta \cdot v \mathrm{~d} \Gamma=0 \quad \forall \theta \in V_{\theta} .
$$

This equation is nothing but the variational form of the well-known Griffith's criterion; it implies $G=G_{\mathrm{c}}$ along the front only if $G$ and $\hat{\gamma}_{f}$ are smooth enough. Eq. (30) is non-linear with respect to the variable $\theta$ and is solved by the Newton's method. At each iteration of the Newton's algorithm, the front displacement $\Theta \in V_{\theta}$ is computed by solving the following variational problem

$$
E^{(2)}(\Theta, \theta)=-E^{(1)}(\theta) \quad \forall \theta \in V_{\theta} .
$$

Let us insist on the point that $E^{(2)}$ is a non-local operator. Consequently, the points of the front are not moved independently. The displacement $\Theta$ is proportional to the value $G-G_{\mathrm{c}}$ not locally on each point of $\hat{\gamma}_{f}$ but in a variational sense. 


\section{Numerical approximation}

\subsection{Displacement and front approximation}

The domain $\hat{\Omega}$ is meshed using 16-noded finite elements for which the shape functions are quadratic with respect to the in-plane variables and linear with respect to the out-of-plane variable. The nodes are repeated on the delaminated part $\hat{\omega}_{\mathrm{d}}$ of $\hat{\omega}$.

The front $\hat{\gamma}_{f}$ is approximated using $B_{3}$-spline functions. This allows us to disconnect the $\hat{\gamma}_{f}$ description from the finite element mesh. The curve $\hat{\gamma}_{f}$ is described by nodes $x^{i}$ and the part of $\hat{\gamma}_{f}$ located between two nodes constitutes a spline element. Then, the parametric equation of the $k$ th element is

$$
x(\tau)=\sum_{i=1}^{4} p_{i}(\tau) \phi_{x}^{i+k-1},
$$

where $\phi_{x}^{i}$ are the geometrical spline coordinates and $\tau$ is the curvilinear abscissa along $\hat{\gamma}_{f}$. Finally, in the neighbourhood of the $k$ th spline element, $\theta$ is defined as

$$
\theta(\tau, \rho)=q(\rho) \sum_{i=1}^{4} p_{i}(\tau) \phi_{\theta}^{i+k-1},
$$

where $\rho$ is the transverse local coordinate to the front defined by the relation $O M=O m+\rho e_{2}$ (see Fig. 2 for notations). $q(\rho)$ is a smooth bell-shaped function equal to 1 for $\rho=0$ and 0 for $\rho=\rho_{r}$. The $\theta$ degrees of freedom are the spline coordinates $\phi_{\theta}$. Generally, their number is lower than the number of all the nodes of the finite element mesh located on $\hat{\gamma}_{f}$.

The discretized form of relations (21) and first term of (24) can be written as $\{\theta\}^{\mathrm{t}} \cdot\{g\}$ and $\{\theta\}^{\mathrm{t}} \cdot\left\{g_{\mathrm{c}}\right\}$ respectively whereas the right-hand side of problem (20) is discretized as:

$$
\{v\}^{\mathrm{t}} \cdot[F] \cdot\{\theta\} .
$$

Finally, the second derivatives (23) and second term of (24) take the following forms:

$$
\{\theta\}^{\mathrm{t}} \cdot\left[J^{(2)}\right] \cdot\{\theta\} ; \quad\{\theta\}^{\mathrm{t}} \cdot\left[D^{(2)}\right] \cdot\{\theta\},
$$

where $\left[J^{(2)}\right]$ and $\left[D^{(2)}\right]$ are symmetric matrices, $\left[J^{(2)}\right]$ being fully populated.

\subsection{Delamination growth algorithm}

The delamination growth algorithm is described in the case of displacement control. Let

- $\left[K_{\mathrm{T}}\right]$ be the tangent stiffness matrix;

- $\{R\}$ be the vector of residual forces;

- $j$ the number of the control degree of freedom;

- $[Q]$ be the matrix of the eigenvectors of $\left[D^{(2)}\right]$ associated to positive eigenvalues.

Then, the algorithm is as follows:

loop $n$ : control displacement increment $u_{\mathrm{d}}^{n}$ loop $k$ : front increment $\gamma_{f}^{n, k}$

loop $i$ : equilibrium equations

$$
\begin{aligned}
& \left\{\Delta u^{i}\right\}=\left\{\Delta u_{\mathrm{R}}^{n, k, i}\right\}+\Delta \lambda^{i}\left\{u_{f}^{n, k}\right\} \\
& {\left[K_{\mathrm{T}}^{n, k}\right] \cdot\left\{\Delta u_{\mathrm{R}}^{n, k}\right\}=-\left\{R^{n, k, i}\right\}}
\end{aligned}
$$




$$
\begin{gathered}
{\left[K_{\mathrm{T}}^{n, k}\right] \cdot\left\{u_{f}^{n, k}\right\}=\{f\}} \\
\Delta \lambda^{i}=-\frac{\left\{\Delta u_{\mathrm{R}}^{n, k, i}\right\}_{j}}{\left\{u_{f}^{n, k}\right\}_{j}} \\
\left\{u^{n, k, i+1}\right\}=\left\{u^{n, k, i}\right\}+\left\{\Delta u^{i}\right\} \\
\lambda^{n, k, i+1}=\lambda^{n, k, i}+\Delta \lambda^{i}
\end{gathered}
$$

if $\left\|\left\{R^{n, k, i+1}\right\}\right\| \leqslant \epsilon_{\mathrm{R}}$, end of loop $i$

compute $\left[K_{\mathrm{T}}^{n, k}\right]$ at the new equilibrium point

compute $\{g\}$ and $\left\{g_{\mathrm{c}}\right\}$

compute $\left[u^{(1)}\right]$ solving $\left[K_{\mathrm{T}}^{n, k}\right] \cdot\left[u^{(1)}\right]=\left[F^{n, k}\right]$

compute $\left[J^{(2)}\right],\left[D^{(2)}\right],[Q]$

compute $\{\Theta\}$ solving

$$
\left([Q]^{\mathrm{t}} \cdot\left[E^{(2)}\right] \cdot Q\right) \cdot\{\Phi\}=[Q]^{\mathrm{t}} \cdot\left\{g-g_{\mathrm{c}}\right\} \quad\{\Theta\}=[Q] \cdot\{\Phi\}
$$

if $\|\{\theta\}\|>\epsilon_{\theta}$ move the front:

$$
\gamma_{f}^{n, k+1}: x^{n, k+1}=x^{n, k}+\epsilon_{\mathrm{S}} \theta\left(x^{n, k}\right)
$$

remeshing and go to loop $i$

if $\|\{\theta\}\| \leqslant \epsilon_{\theta}$ end of loop $k$

$$
u_{\mathrm{d}}^{n+1}=u_{\mathrm{d}}^{n}+\Delta u_{\mathrm{d}}^{n}
$$

end of loop $n$.

This algorithm can be repeated until $\hat{\gamma}_{f} \subset \partial \hat{\omega}$, i.e. until the total separation of the lower and upper parts of $\hat{\Omega}$. We are now going to make some comments with regard to this algorithm:

1. The matrix $[Q]$ introduced in (34) is composed of the eigenvectors associated to non-negative eigenvalue of $\left[D^{(2)}\right]$. This ensures that the displacement field $\Theta$ verify $D^{(2)}(\Theta, \Theta) \geqslant 0$.

2. Nodal values of the delamination force (vector $\{G\}$ ) are not needed. However, if $\{G\}_{m}<G_{\mathrm{c}} \forall m$ then the load is not large enough and (34) will give $\{\Theta\}_{p}<0 \forall p$. In conclusion, the knowledge of $\{G\}$ can avoid unuseful computations (for the detail of computation of $\{G\}$, see [2]).

3. There are several ways to compute the new displacement field after remeshing. Here, we have presented the case where the new equilibrium point is sought starting from the displacements obtained with the previous mesh. Unfortunately, if the front displacement is too large, the equilibrium cannot be recovered. To prevent divergence, small increments of the front displacement must be made, prescribing a maximum value $\Theta_{\max }$ of $\Theta$. Another way is to restart the computation from unloading. This way seems more costly, but it allows greater displacement front increments.

4. The remeshing step can be time consuming for complex structures. Some authors have recently proposed a method without remeshing where the description of the crack front is independent of the mesh structure $[33,34]$.

5. $\epsilon_{\theta}$ and $\Theta_{\max }$ can be taken as a fraction of a characteristic length of $\hat{\omega}$. For numerical application, we have taken $\epsilon_{\mathrm{S}}=\min \left(1, \Theta_{\max } /\left(\|\{\Theta\}\| L^{\infty}\right)\right)$.

\section{Numerical applications}

\subsection{Analytical solution for the beam problem}

The first application concerned a curved beam of constant thickness $\epsilon$ and of constant radius of curvature $r$. The beam was made of an isotropic homogeneous material and had a crack along its mid-axis (see Fig. 3). 


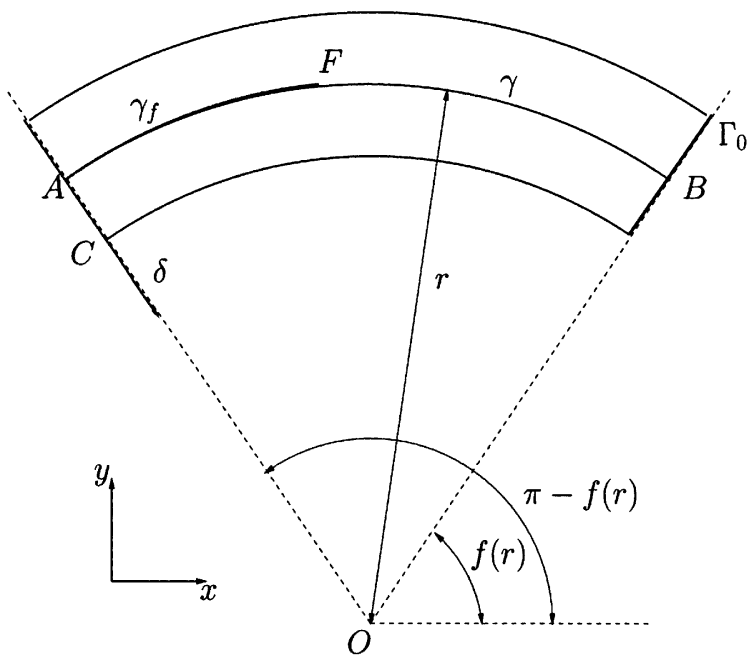

Fig. 3. Cylindrical delaminated arch.

It was clamped at the cross-section containing the point $B$ and was submitted to a normal displacement $\delta$ at the end of the lower delaminated arm (point $C$ ). The mid-axis $\gamma$ was described by the injective mapping $\phi: \zeta_{1} \in[f(r), \pi-f(r)] \rightarrow(x, y) \in \gamma$ defined as follows

$$
\phi\left(\zeta_{1}\right)=\left(\frac{l}{2}+r \cos \zeta_{1},-\sqrt{r^{2}-\frac{l^{2}}{4}}+r \sin \zeta_{1}\right), \quad f(r)=\frac{1}{2}\left(\pi-\frac{l}{r}\right),
$$

for which the length $l$ of $\gamma$ is independent of $r$. The mechanical energy derivatives were obtained analytically for $r$ sufficiently large [24] and are recalled here

$$
\begin{aligned}
& J(a, r)=f_{1}(a)-\frac{f_{2}(a)}{r^{2}}+\mathrm{O}\left(\frac{1}{r^{4}}\right), \quad f_{1}(a)=\frac{E \epsilon^{3} \delta^{2}}{l^{3}+7 a^{3}}, \quad f_{2}(a)=-\frac{1}{8} \frac{l^{5} E \epsilon^{3} \delta^{2}}{\left(l^{3}+7 a^{3}\right)^{2}}, \\
& g(a, r)=-f_{1}^{\prime}(a)-\frac{-f_{2}^{\prime}(a)}{r^{2}}+\mathrm{O}\left(\frac{1}{r^{4}}\right), \quad J^{(2)}(a, r)=f_{1}^{\prime \prime}(a)-\frac{f_{2}^{\prime \prime}(a)}{r^{2}}+\mathrm{O}\left(\frac{1}{r^{4}}\right),
\end{aligned}
$$

where $a$ is the crack length.

Observing that the functions $f_{i}^{\prime}(a)$ are negative, the sign of $J^{(2)}$ can be determined easily. It is reported in Table 1 where $C$ is the curvature of the beam.

The interest of this example is twofold:

- Firstly, it shows that the energy release rate is a decreasing function of the curvature. This implies that the curvature is a limiting factor for the crack growth.

- Secondly, it shows that the curvature has an influence on the growth stability given by the sign of $J^{(2)}$.

Table 1

Sign of $J^{(2)}$

\begin{tabular}{llll}
\hline & $a_{1}$ & $a_{2}$ & $l$ \\
\hline$C^{2}>f_{1}^{\prime \prime}(a) / f_{2}^{\prime \prime}(a)$ & + & - & - \\
$0 \leqslant C^{2}<f_{1}^{\prime \prime}(a) / f_{2}^{\prime \prime}(a)$ & - & - & + \\
\hline$a_{1}=\left(\frac{2}{49}\right)^{1 / 3} l, a_{2}=\left(\frac{1}{14}\right)^{1 / 3} l$. & &
\end{tabular}




\subsection{Cylindrical arch}

The second example was devoted to the study of a cylindrical arch of constant thickness $h$, of constant radius of curvature $r$, of width $L$ and of length $l$ (see Fig. 4).

It was made of an isotropic homogeneous material and had a delamination in its mid-plane. The geometrical and the mechanical characteristics of the arch are reported in Table 2.

The arch was described by the mapping $\varphi[35]$

$$
\Omega \ni(x, y, z)=\varphi\left(\zeta_{1}, \zeta_{2}, \zeta_{3}\right)=\left(\left(r+\zeta_{3}\right) \sin \left(\frac{\zeta_{1}}{r}\right), \zeta_{2},\left(r+\zeta_{3}\right) \cos \frac{\zeta_{1}}{r}-r \cos \frac{l}{2 r}\right), \quad\left(\zeta_{1}, \zeta_{2}, \zeta_{3}\right) \in \hat{\Omega},
$$

defined on the domain $\hat{\Omega}=]-l / 2, l / 2[\times] 0, L[\times]-h, h[$ with $r$ satisfying the following condition of injectivity

$$
r>\max \left(h, \frac{l}{2 \pi}\right) .
$$

For such a mapping, the covariant basis was

$$
\vec{a}_{1}=\left(\cos \frac{\zeta_{1}}{r}, 0,-\sin \frac{\zeta_{1}}{r}\right), \quad \vec{a}_{2}=(0,1,0), \quad \vec{a}_{3}=\left(\sin \frac{\zeta_{1}}{r}, 0, \cos \frac{\zeta_{1}}{r}\right),
$$

leading to the following expressions (see Appendix A for the definitions):

$$
\underline{d}(v)=\left(\begin{array}{ccc}
1+\zeta_{3} r^{-1} & 0 & 0 \\
0 & 1 & 0 \\
0 & 0 & 1
\end{array}\right)^{-1}, \quad f(v)=\left(\begin{array}{ccc}
-r^{-1} v_{3} & 0 & -r^{-1} v_{1} \\
0 & 0 & 0 \\
0 & 0 & 0
\end{array}\right)
$$

As $\theta_{3}=0$ and as the tensors $a$ and $\underline{S}$ and the operators $\underline{d}$ and $f$ are independent of the variables $\zeta_{\alpha}$, we have $(\nabla \underline{d} \cdot \theta)_{i j}=0,(\nabla \underline{f} \cdot \theta)_{i j}=0,(\nabla \underline{S} \cdot \theta)_{i j}=0$ and $(\nabla a \cdot \theta)_{i j}=0$. The derivatives of the fracture energy could be written as:

$$
D^{(1)}(\theta)=-G_{\mathrm{c}} \int_{\mathrm{c}_{\hat{\omega}_{\mathrm{d}}}}\left(1+\frac{\zeta_{3}}{r}\right) \operatorname{div} \theta \mathrm{d} \hat{\omega}_{0} ; \quad D^{(2)}(\theta, \theta)=-G_{\mathrm{c}} \int_{\mathrm{c}_{\hat{\omega}_{\mathrm{d}}}}\left(1+\frac{\zeta_{3}}{r}\right) \operatorname{det}(\nabla \theta) \mathrm{d} \hat{\omega}
$$
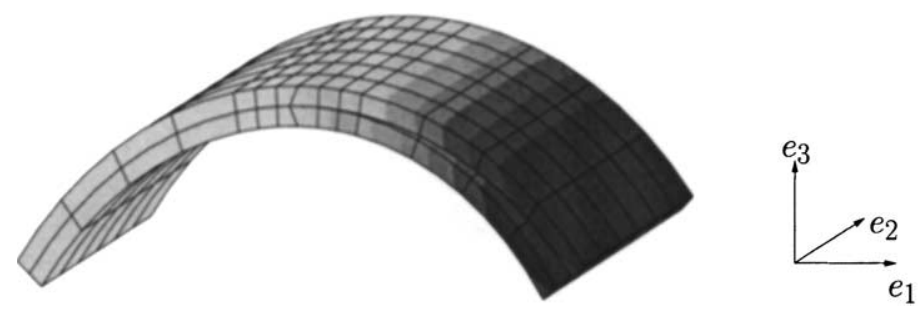

Fig. 4. Cylindrical arch.

Table 2

Material and geometrical characteristics

\begin{tabular}{llllllll}
\hline$E$ & $v$ & $G_{\mathrm{c}}$ & $l$ & $L$ & $h$ & $\Theta_{\max }$ & $\epsilon_{\theta}$ \\
\hline $126,000 \mathrm{MPa}$ & 0.3 & $0.281 \mathrm{~N} / \mathrm{mm}$ & $100 \mathrm{~mm}$ & $100 \mathrm{~mm}$ & $2.5 \mathrm{~mm}$ & $10^{-2} l(\mathrm{~mm})$ & $4 \times 10^{-4} l(\mathrm{~mm})$ \\
\hline
\end{tabular}


so that the spectrum of $D^{(2)}$ was symmetric with respect to zero (see Appendix B for the discussion on the spectrum).

Two cases were studied: the case of an opening delamination and the case of an internal delamination.

\subsubsection{Opening delamination}

The arch had an initial delamination with a straight front $\hat{\gamma}_{f}=\left\{\zeta \in \hat{\Omega}, \zeta_{1}=l / 4, \zeta_{3}=0\right\}$. It was clamped on the side $\Gamma_{u}=\left\{\zeta \in \hat{\Omega}, \zeta_{1}=-l / 2\right\}$ and submitted to a normal load $\hat{f}=\left(0,0, \hat{f}_{3}^{ \pm}\right), \hat{f}_{3}^{+}=-\hat{f}_{3}^{-}=8 \mathrm{~N}$ on the opposite sides. The modified Newton's algorithm was displacement controlled; let $u_{\mathrm{d}}$ be the normal displacement at the end of the upper delaminated arm (the control variable). Figs. 5 and 6 depict the variations of the loading factor and of the delamination length respectively as a function of $u_{\mathrm{d}}$ for two values of $r$.

Fig. 5 shows that the curvature increases slightly the stiffness of the arch. The points of the same abscissa correspond to the iterations over $\theta$ (loop $k$ of the present algorithm); only the lower points ensure in addition the minimum of $E$ with respect to the front location. The convergence of the front location is obtained in six iterations with an increment $\delta u_{\mathrm{d}}=0.025 \mathrm{~mm}$ and in 12 iterations with $\delta u_{\mathrm{d}}=0.1 \mathrm{~mm}$. The different equilibrium points obtained with the two values of $\delta u_{\mathrm{d}}$ are plotted in Fig. 7. They are on the same curve.

Fig. 8 depicts the evolution of the energy release rate as the radius of curvature of the arch is increasing. It is found that, in accordance with remark made for the analytical example, the curvature is a limiting factor for the crack growth. Finally, the front locations at arrest for different values of $u_{\mathrm{d}}$ are plotted in Fig. 9. For convenience, the front shapes are represented in the reference plane $\left(\zeta_{1}, \zeta_{2}\right)$.

As the specimen is wider than longer, the edge effects have a weak and local influence on the front shapes that are straight, except in the vicinity of the lateral edges [36]. Finally, let us mention that the growth was stable as all the eigenvalues of $\left[J^{(2)}\right]$ were positive.

\subsubsection{Internal delamination}

Then, the case of an internal delamination with a circular front shape (in the reference plane) $\hat{\gamma}_{f}=\left\{\zeta_{1}^{2}+\zeta_{2}^{2}=a^{2}, \zeta_{3}=0\right\}$ centred at the origin was considered. $a$ is the radius of the front and is equal to

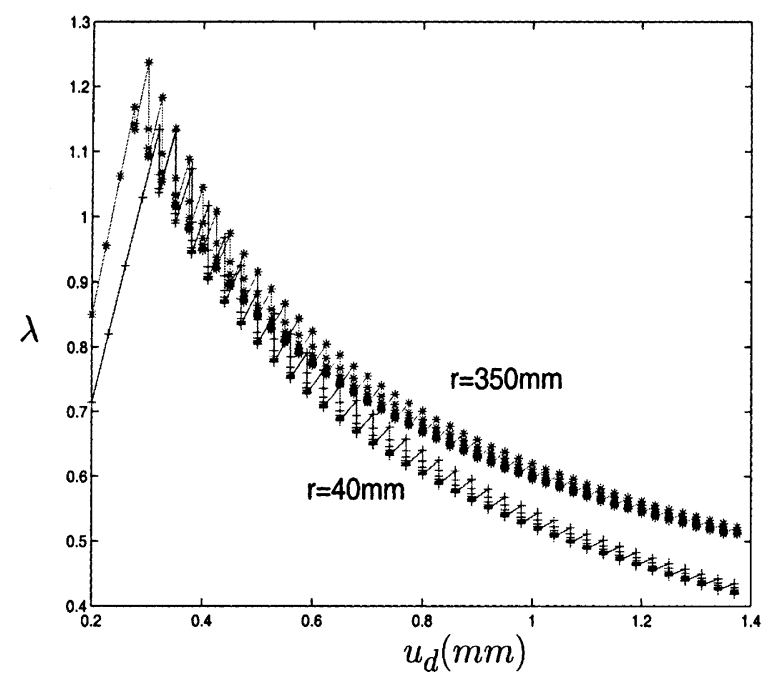

Fig. 5. Load factor vs. $u_{\mathrm{d}}$. 


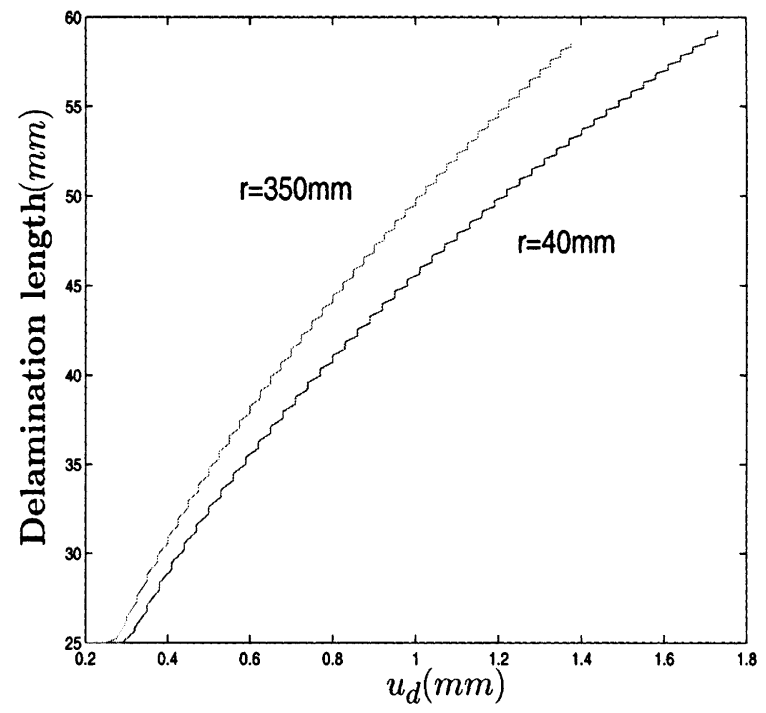

Fig. 6. Delamination lengths vs. $u_{\mathrm{d}}$.

$40 \mathrm{~mm}$. The arch was clamped on the sides $\Gamma_{u}=\left\{\zeta_{1}= \pm l, \zeta_{2}=0, L\right\}$ and was submitted to two opposite pointwise normal loads $\hat{f}_{3}^{+}=-\hat{f}_{3}^{-}=100 \mathrm{~N}$ applied at the centres of the delamination. The characteristics of the arch were unchanged, except $L=200 \mathrm{~mm}$. As, in the previous case, the modified Newton's algorithm was displacement controlled, the control variable being the normal displacement $u_{\mathrm{d}}$ of the upper delamination centre. Fig. 10 shows the initial mesh and the deformed mesh after delamination growth.

Figs. 11 and 12 depict the variations of the loading factor and the distance to the origin of the points A and B (defined in Fig. 10) of the front respectively as a function of $u_{\mathrm{d}}$, for two different values of the radius of curvature. They show firstly, that a higher value of $u_{\mathrm{d}}$ was needed to make the delamination grow in the

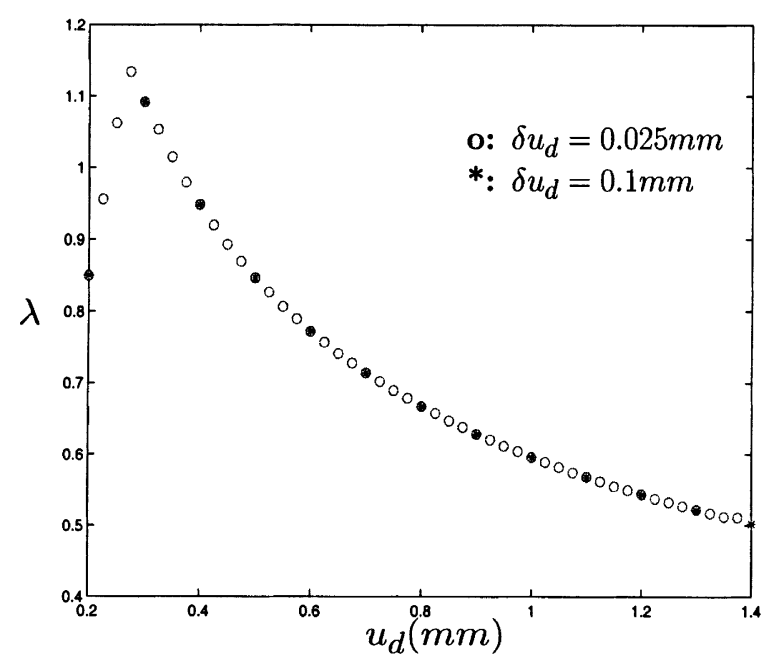

Fig. 7. Equilibrium curve $\left(u_{\mathrm{d}}, \lambda\right)$. 


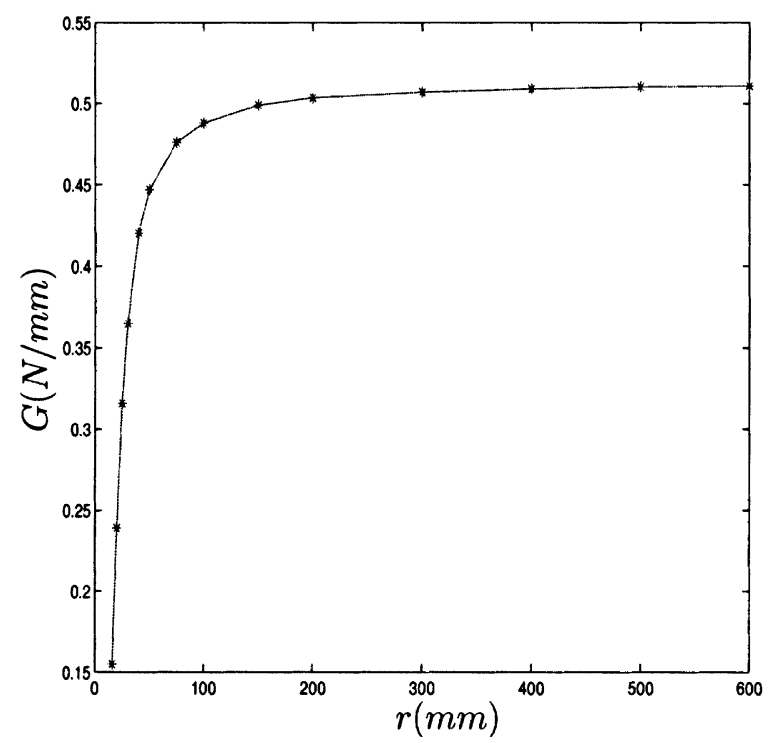

Fig. 8. Delamination force vs. $r$.

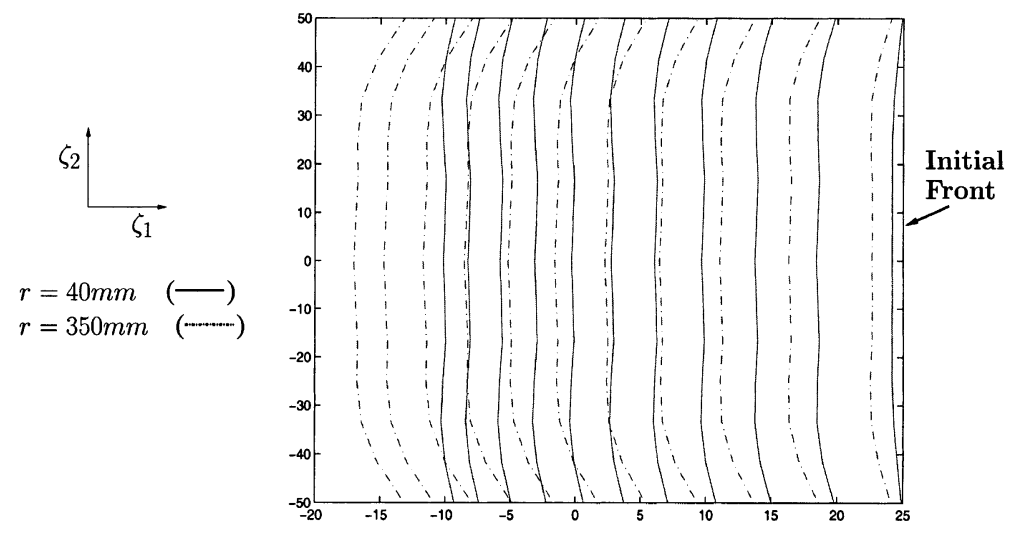

Fig. 9. Front positions associated to $u_{\mathrm{d}}=0.32,0.47,0.62,0.77,0.92,1.07,1.22,1.37,1.52,1.67,1.79$ (mm).

most curved case and secondly, that the crack was growing faster along the $e_{2}$-axis where the curvature is minimal (point B).

The different front locations are plotted in Figs. 13 and 14 for $r=300$ and $900 \mathrm{~mm}$ respectively, showing that the front shapes remain symmetric with respect to the axes during the delamination growth.

The values of the local energy release rate $G$ and its associated normal front displacement along the part of the front $\left(\zeta_{1}>0, \zeta_{2}>0\right)$ are reported in Fig. 15. The front displacement appears, up to a multiplying constant, to be a regularisation of the quantity $G-G_{\mathrm{c}}$. The variations of the local energy release rate at the points $\mathrm{A}$ and $\mathrm{B}$ with respect to the radius of curvature are reported in Fig. 16. As in the previous cases, a similar variation of the form $K_{1}-\left(K_{2} / r^{2}\right)$ was obtained, reaching the same value when $r$ tends to infinity.

To conclude, we are going to make some comments on the growth stability. The computations made for the two values of the radius of curvature $(r=300$ and $900 \mathrm{~mm})$, the results of which are reported here, showed a stable delamination growth. However, it can be observed in Figs. 11 and 12 that, for the smaller 


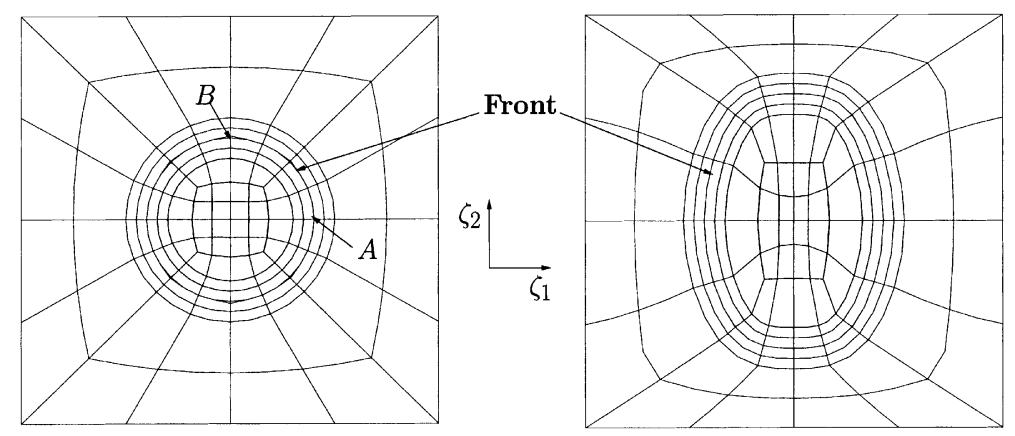

Fig. 10. Initial mesh (left) and ended mesh for $r=300 \mathrm{~mm}$ and $u_{\mathrm{d}}=4.62 \mathrm{~mm}$ (right).

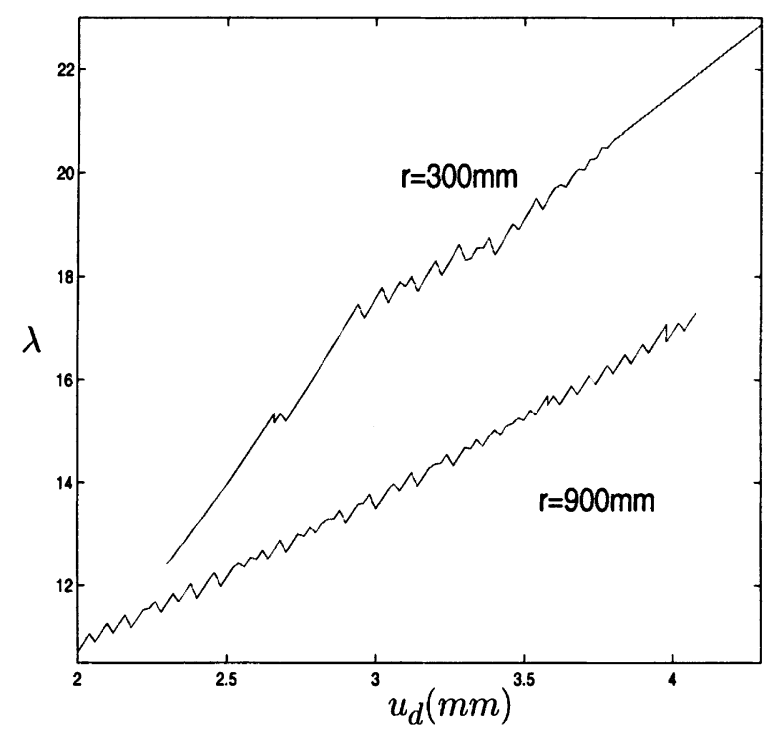

Fig. 11. Load factor vs. $u_{\mathrm{d}}$.

values of $r$, the arch response is irregular for a prescribed normal displacement less than $3.75 \mathrm{~mm}$. Computations made for a radius of $200 \mathrm{~mm}$ revealed an unstable growth.

\section{Conclusion}

We have explained an algorithm for the numerical simulation of delamination growth in a curved interface. It proved to be robust and fast as the convergence in $\theta$ was obtained in less than 10 iterations in most cases. Moreover, it can be developed independently as a post-processor of a standard finite elements code. However, it requires the coupling between a code of analysis and a meshing code. The necessity to make a re-meshing, at least in the neighbourhood of the front when it is moved, can become a hard task for complex structures.

Two questions remain unanswered 


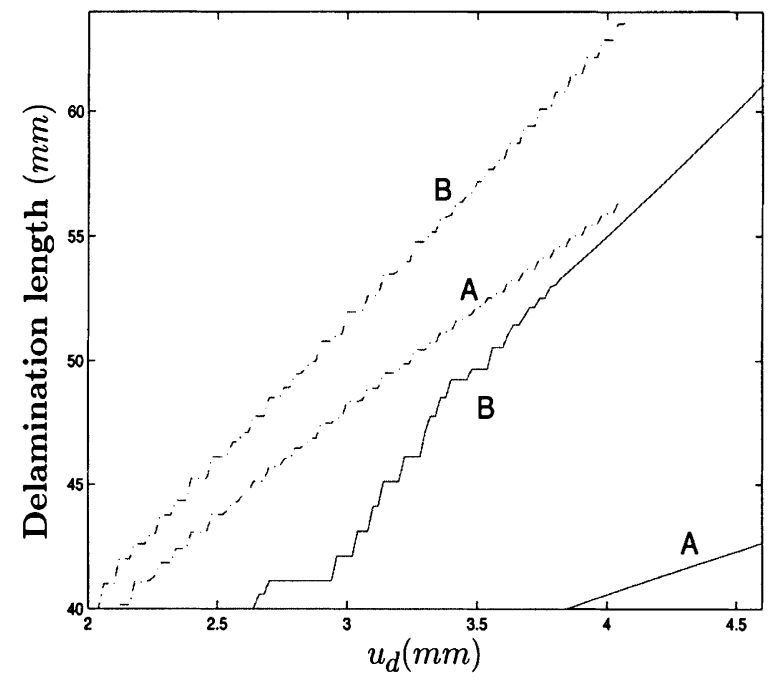

Fig. 12. Delamination lengths vs. $u_{\mathrm{d}}: r=300 \mathrm{~mm} \mathrm{(-)}$ and $r=900 \mathrm{~mm}(-)$.

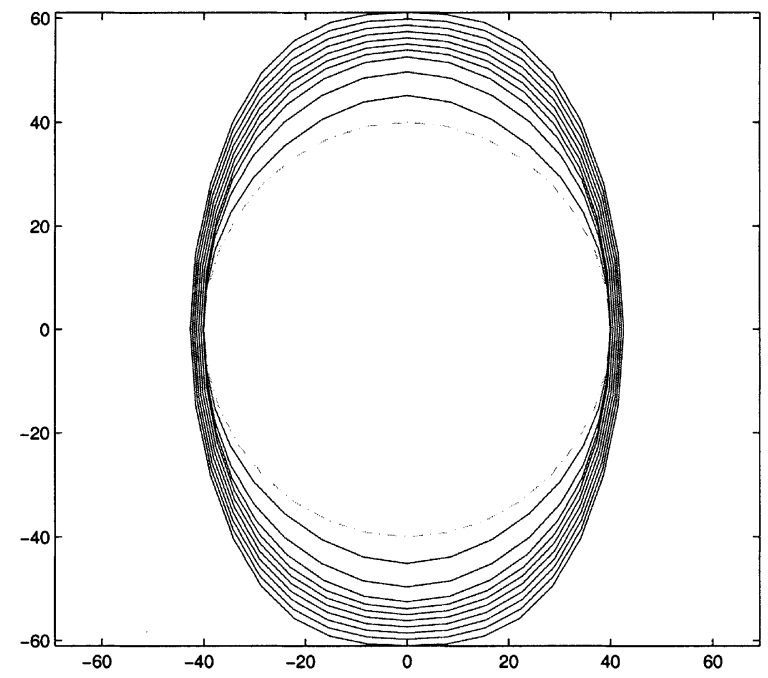

Fig. 13. Front positions for $r=300 \mathrm{~mm}$ associated to $u_{\mathrm{d}}=3.22,3.56,3.78,3.9,4.02,4.14,4.26,4.38,4.5,4.62$ (mm).

- The first concerns the way to restore the equilibrium after the front has been moved. In the examples presented here, only the internal loop in the algorithm was made in order to improve the displacement field obtained with the previous mesh. This way requires to make small increments of the front displacement to be able to determine the new equilibrium point. However, there are cases for which it is not sufficient as it was observed studying a stiffened panel loaded in compression [3] where it was binding to restart the computation from unloading. This way allows to make greater front displacement increments.

- The second concerns the $\left[J^{(2)}\right]$ spectrum, or more precisely the spectrum of the projection $[Q]^{\mathrm{t}} \cdot\left[J^{(2)}\right] \cdot[Q]$ of $\left[J^{(2)}\right]$ onto the subspace spanned by the eigenvectors associated to the positive eigenvalues of $\left[D^{(2)}\right]$. 


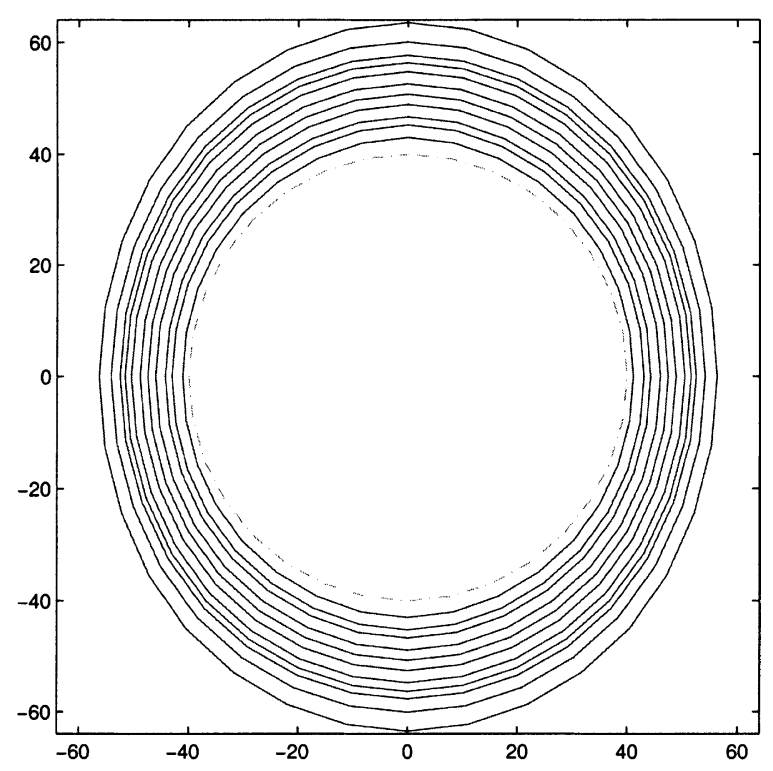

Fig. 14. Front positions for $r=900 \mathrm{~mm}$ associated to $u_{\mathrm{d}}=2.26,2.46,2.6,2.8,2.96,3.14,3.32,3.46,3.58,3.8,4.08$ (mm).

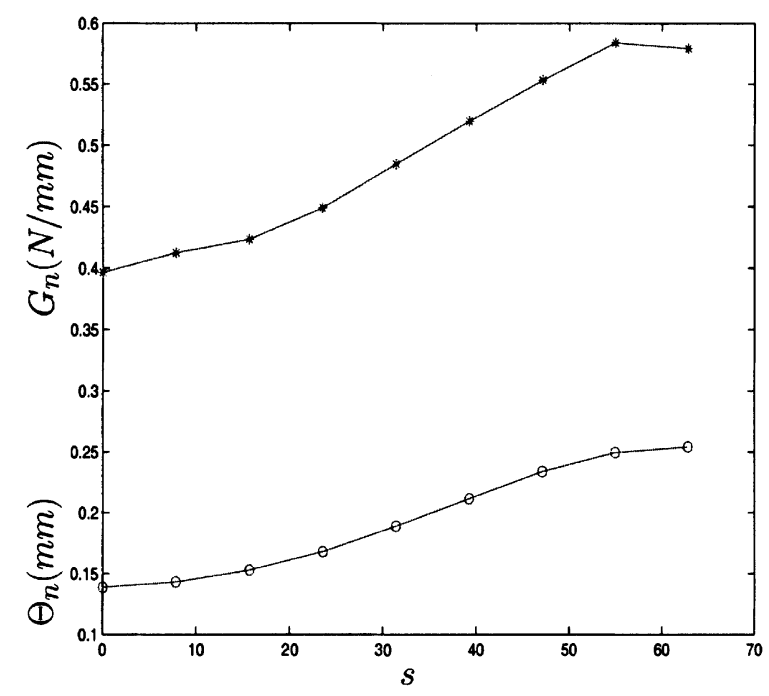

Fig. 15. Normal delamination force (*) and normal displacement (o) along $\hat{\gamma}_{f}: r=900 \mathrm{~mm}, u_{\mathrm{d}}=2.7 \mathrm{~mm}$.

When, the growth is stable, all the eigenvalues are positive, and negative when it is unstable. However, we have encountered case for which there was positive and negative eigenvalues. We have observed, in the cases of stable growth, that the number of negative eigenvalues was decreasing as the number of front displacements was increasing. We speculate that these negative eigenvalues are related to the fact that the initial front shape was not acceptable i.e.: the shape of the artificial initial defect does not correspond to the shape of the delamination that would appear under the applied loading. There is then first an unstable growth to reach the natural delamination front shape. 


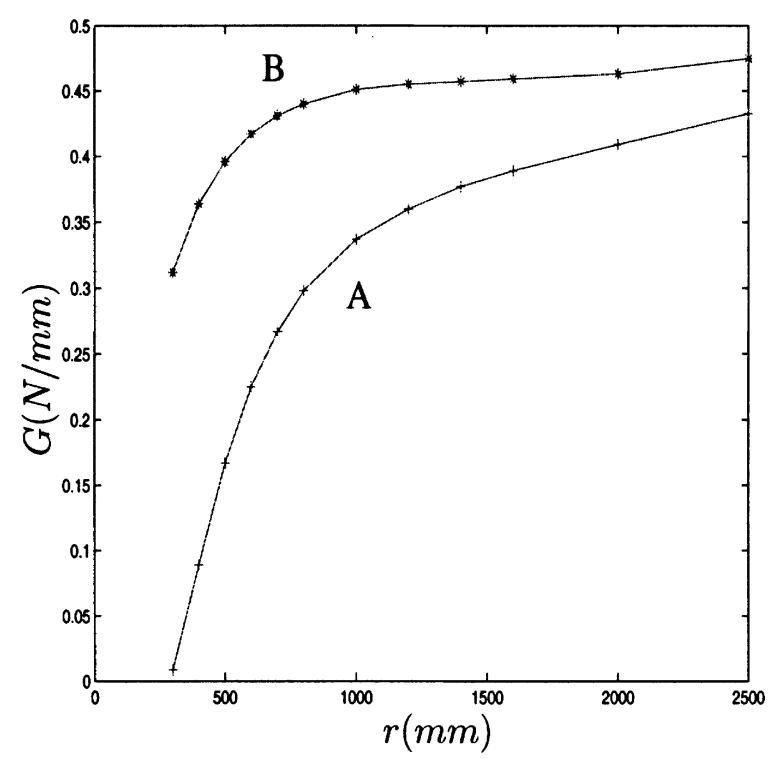

Fig. 16. Delamination force vs. $r: u_{\mathrm{d}}=2.3 \mathrm{~mm}$.

This last point will have to be detailed in the future.

\section{Appendix A. Expression of the variational formulation (5) in the local reference frame}

Let us consider a vector $v$ expressed in the contravariant basis $v=v_{\alpha} a^{\alpha}+v_{3} a^{3}$. For all points $m$ on $S$, a straight computation leads to

$$
\frac{\partial v}{\partial m}=\left(v_{\beta \mid \alpha}-b_{\alpha \beta} v_{3}\right) a^{\alpha} \otimes a^{\beta}+\left(v_{3, \alpha}+b_{\alpha}^{\beta} v_{\beta}\right) a^{\alpha} \otimes a^{3},
$$

where $v_{\alpha \mid \beta}=v_{\alpha, \beta}-\Gamma_{\alpha \beta}^{\gamma} v_{\gamma}, b_{\alpha}^{\beta}=b_{\alpha \lambda} a^{\lambda \beta}$ and $\Gamma_{\beta \gamma}^{\alpha}=a^{\alpha} \cdot a_{\gamma, \beta}$ the Christoffel symbols.

Let us now note the covariant basis $g_{i}$ associated to any point $x$ of $\Omega$ by $g_{\alpha}=\left(\delta_{\alpha}^{\gamma}-\zeta_{3} b_{\alpha}^{\gamma}\right) a_{\gamma}$ and $g_{3}=a_{3}$, the covariant components of the metric tensor $g_{i j}=g_{i} \cdot g_{j}$, the contravariant basis $g^{i}=g^{i j} g_{j}$ where $g^{i j}=$ $\left(g_{i j}\right)^{-1}$. Then for any point $M \in \Omega$, we have

$$
\frac{\partial v}{\partial M}=\left(v_{\beta \mid \alpha}-b_{\alpha \beta} v_{3}\right) g^{\alpha} \otimes a^{\beta}+\left(v_{3, \alpha}+b_{\alpha}^{\beta} v_{\beta}\right) g^{\alpha} \otimes a^{3}+v_{\alpha, 3} g^{3} \otimes a^{\alpha}+v_{3,3} g^{3} \otimes a^{3},
$$

and the relation $g^{\alpha}=\left(\delta_{\alpha}^{\beta}-\zeta_{3} b_{\alpha}^{\beta}\right)^{-1} a^{\beta} \equiv d_{\beta}^{\alpha} a^{\beta}$ gives

$$
\operatorname{Tr}\left(\sigma \cdot \frac{\partial v}{\partial M}\right)=\sigma_{i j} v_{j, i}=\sigma^{i j} d_{j}^{\tau} e_{\tau i}(v) ; \quad \operatorname{Tr}\left(\sigma \cdot \frac{\partial u^{\mathrm{t}}}{\partial M} \cdot \frac{\partial v}{\partial M}\right)=\sigma^{i j} d_{m}^{\tau} e_{\tau i}(\hat{u}) d_{n}^{\gamma} e_{\gamma j}(v) a^{m n}
$$

where

$$
e_{\tau i}(v)= \begin{cases}v_{\beta, \alpha}-\Gamma_{\alpha \beta}^{\gamma} v_{\gamma}-b_{\alpha \beta} v_{3} & (\tau, i)=(\alpha, \beta), \\ v_{3, \alpha}+b_{\alpha}^{\gamma} v_{\gamma} & (\tau, i)=(\alpha, 3), \\ v_{\alpha, 3} & (\tau, i)=(3, \alpha), \\ v_{3,3} & (\tau, i)=(3,3)\end{cases}
$$


If we define the operator $\hat{d}$ such that $\hat{d}_{i j}=d_{i}^{j}$ and the operator $f$ such that

$$
f(v)=\left(\begin{array}{ccc}
-\left(\Gamma_{11}^{\gamma} v_{\gamma}+b_{11} v_{3}\right) & -\left(\Gamma_{12}^{\gamma} v_{\gamma}+b_{12} v_{3}\right) & b_{1}^{\gamma} v_{\gamma} \\
-\left(\Gamma_{21}^{\gamma} v_{\gamma}+b_{21} v_{3}\right) & -\left(\Gamma_{22}^{\gamma} v_{\gamma}+b_{22} v_{3}\right) & b_{2}^{\gamma} v_{\gamma} \\
0 & 0 & 0
\end{array}\right)
$$

then we have $e_{i j}(v)=v_{j, i}+f_{i j}(v)$ and finally the following relations

$$
\begin{aligned}
& \operatorname{Tr}\left(\sigma(x) \cdot F^{\mathrm{t}}(u(x)) \cdot \nabla v(x)\right)=\operatorname{Tr}\left(\hat{\sigma}(\zeta) \cdot \hat{F}(\hat{u}(\zeta)) \cdot(\hat{d} \cdot e(v))^{\mathrm{t}}\right) ; \quad \hat{F}(\hat{u})=I_{3}+\hat{d} \cdot e(\hat{u}) \cdot a, \\
& \operatorname{Tr}(\tau(x) \cdot \gamma(u(x)))=\operatorname{Tr}(\hat{\tau}(\zeta) \cdot \hat{\gamma}(\hat{u}(\zeta))) ; \quad \hat{\gamma}(\hat{u}(\zeta))=\frac{1}{2}\left(\hat{d} \cdot e(\hat{u})+(\hat{d} \cdot e(\hat{u}))^{\mathrm{t}}+\hat{d} \cdot e(\hat{u}) \cdot a \cdot(\hat{d} \cdot e(\hat{u}))^{\mathrm{t}}\right),
\end{aligned}
$$

where $a$ is here the contravariant metric tensor $a=\left(a^{i j}\right), a^{\alpha \beta}=a^{\alpha} \cdot a^{\beta}, a^{i 3}=\delta^{i 3}$. Finally, the variational formulation (5) becomes on $\hat{S}$

$$
\begin{cases}\int_{\hat{\Omega}} \operatorname{Tr}(\hat{S}: \hat{\sigma} \cdot \hat{\tau}) \sqrt{|g|}=\int_{\hat{\Omega}} \operatorname{Tr}(\hat{\tau} \cdot \hat{\gamma}(\hat{u})) \sqrt{|g|} & \forall \hat{\tau} \in \Sigma(\hat{\Omega}), \\ \int_{\hat{\Omega}} \operatorname{Tr}\left(\hat{\sigma} \cdot \hat{F} \cdot(\hat{d} \cdot e(\hat{v}))^{\mathrm{t}}\right) \sqrt{|g|}=\lambda \int_{\hat{\Gamma}_{f}} \hat{t} \cdot \hat{v} \sqrt{|g|} \mathrm{d} \Gamma & \forall \hat{v} \in V \in V(\hat{\Omega}),\end{cases}
$$

where $V(\hat{\Omega})=\left\{v \in\left(W^{1,4}(\hat{\Omega})\right)^{3} / v_{\mid \hat{\Gamma}_{u}}\right\}$ and $\Sigma(\hat{\Omega})=\left(L_{\mathrm{s}}^{2}(\hat{\Omega})\right)^{9}, \hat{f}=f^{i} \vec{a}_{i}, f^{i}$ the contravariant components of $f(|g|)^{1 / 2}$ is a positive scalar

$$
\sqrt{|g|}=\sqrt{\operatorname{det}\left(g_{\alpha \beta}\right)}=\left(1-2 H \zeta_{3}+K \zeta_{3}^{2}\right) \sqrt{\operatorname{det}\left(a_{\alpha \beta}\right)}
$$

where $H$ and $K$ are the mean and the Gauss curvatures respectively [30]. In the case of an isotropic material, the local components of the stiffness tensor $\hat{R}$ associated to the tensor $\hat{S}$ are given by

$$
\hat{R}^{i j k l}\left(\zeta_{\alpha}\right)=\lambda a^{i j}\left(\zeta_{\alpha}\right) a^{k l}\left(\zeta_{\alpha}\right)+\mu\left(a^{i k}\left(\zeta_{\alpha}\right) a^{j l}\left(\zeta_{\alpha}\right)+a^{i l}\left(\zeta_{\alpha}\right) a^{j k}\left(\zeta_{\alpha}\right)\right) .
$$

In order to simplify the application of the $\theta$-method, we define the tensor $\underline{\sigma} \equiv \hat{\sigma} \sqrt{|g|}, \underline{\tau} \equiv \hat{\tau} \sqrt{|g|}$ and $\underline{S} \equiv \hat{S} \sqrt{|g|}^{-1}, \underline{d} \equiv \hat{d}, \underline{F}=\hat{F}, \underline{u}=\hat{u}$. Then, the former formulation becomes

$$
\begin{cases}\int_{\hat{\Omega}} \operatorname{Tr}(\underline{S}: \underline{\sigma} \cdot \underline{\tau})=\int_{\hat{\Omega}} \operatorname{Tr}(\underline{\tau} \cdot \underline{\gamma}(\underline{u})) & \forall \underline{\tau} \in \Sigma(\hat{\Omega}), \\ \int_{\hat{\Omega}} \operatorname{Tr}\left(\underline{\sigma} \cdot \underline{F} \cdot(\underline{d} \cdot e(\hat{v}))^{\mathrm{t}}\right)=\lambda \int_{\hat{\Gamma}_{f}} \hat{t} \cdot \hat{v} \sqrt{|g|} \mathrm{d} \Gamma & \forall \hat{v} \in V(\hat{\Omega}) .\end{cases}
$$

\section{Appendix B. Spectrum of the operator $D^{(2)}$}

For convenience, we note $k=(|g|)^{1 / 2}$. Let us note $s \in[0, l]$ the curvilinear abscissa along the front $\hat{\gamma}_{f}$ and $\rho$ the transverse local coordinate to the front. The vector $\theta$ is expressed along the front as follows:

$$
\theta=\theta_{1} a^{1}+\theta_{2} a^{2}=\theta_{t} \bar{\tau}+\theta_{v} \bar{v}
$$

Then, thanks to the relation $\left(\partial k /\left.\partial x\right|_{\hat{\gamma}_{f}}\right)=k_{s, s} \bar{\tau}+k_{, \rho} \bar{v}$, and the relation $\operatorname{div} \theta \theta \cdot v-v \cdot \nabla \theta \cdot \theta=\left(\partial \theta_{1} / \partial s\right) \theta_{2}-$ $\left(\partial \theta_{2} / \partial s\right) \theta_{1}$, the second term of relation (25) becomes

$$
D^{(2)}(\theta, \theta)=\frac{1}{2} G_{\mathrm{c}} \int_{\hat{\gamma}_{f}} k\left(\frac{\partial \theta_{1}}{\partial s} \theta_{2}-\frac{\partial \theta_{2}}{\partial s} \theta_{1}\right) \mathrm{d} \Gamma+\frac{1}{2} G_{\mathrm{c}} \int_{\hat{\gamma}_{f}}\left(k_{, s} \theta_{\tau}+k_{, \rho} \theta_{v}\right) \theta_{v} \mathrm{~d} \Gamma .
$$


Then, since $D^{(2)}($,$) is a symmetric bilinear form, the use of the relation$

$$
D^{(2)}(\theta, \phi)=\frac{1}{4}\left(D^{(2)}(\theta+\phi, \theta+\phi)-D^{(2)}(\theta-\phi, \theta-\phi)\right),
$$

gives

$$
\begin{aligned}
D^{(2)}(\theta, \phi)= & \frac{1}{2} G_{\mathrm{c}} \int_{\hat{\gamma}_{f}} k\left(\frac{\partial \theta_{1}}{\partial s} \phi_{2}-\frac{\partial \theta_{2}}{\partial s} \phi_{1}\right) \mathrm{d} \Gamma+\frac{1}{2} G_{\mathrm{c}} \int_{\hat{\gamma}_{f}}\left(k_{, s} \theta_{\tau}+k_{, \rho} \theta_{v}\right) \phi_{v} \mathrm{~d} \Gamma \\
& +\frac{1}{4} G_{\mathrm{c}}\left[k\left(\theta_{v} \phi_{\tau}-\theta_{\tau} \phi_{v}\right)\right]_{\mid \hat{\partial \hat{\gamma}_{f}}} \cdot
\end{aligned}
$$

The last term is equal to zero. If $\hat{\gamma}_{f}$ is an open front (Fig. 2 left), this is due to the condition (16). If $\hat{\gamma}_{f}$ is internal (Fig. 2 right), this is due to the fact that $\theta$ at $\partial \hat{\gamma}_{f}: \theta(s=0,0)=\theta(s=l, 0)$. Then, the elements $\lambda$ of the spectrum of $D^{(2)}$ are characterised by the relation

$$
D^{(2)}(\theta, \phi)=\lambda(\theta, \phi)=\lambda \int_{\hat{\gamma}_{f}} \theta \cdot \phi \mathrm{d} s .
$$

They are then solutions of the following parabolic partial differential system $\left(\Lambda=2 G_{\mathrm{c}}^{-1} \lambda\right)$ :

$$
\left(\begin{array}{cc}
0 & -k \\
k & 0
\end{array}\right)\left(\begin{array}{c}
\partial \theta_{1} / \partial s \\
\partial \theta_{2} / \partial s
\end{array}\right)+\left(\begin{array}{cc}
k_{\zeta_{1}} v_{1}-\Lambda & k_{\zeta_{2}} v_{1} \\
k_{\zeta_{1}} v_{2} & k_{\zeta_{2}} v_{2}-\Lambda
\end{array}\right)\left(\begin{array}{l}
\theta_{1} \\
\theta_{2}
\end{array}\right)=\left(\begin{array}{l}
0 \\
0
\end{array}\right)
$$

The characteristic polynomial $p$ is obtained with the choice $\theta=e^{\zeta s} \hat{\theta}, \zeta \in \mathbb{C}$. Let us remark that the condition $\theta(s=0,0)=\theta(s=l, 0)$ implies $\zeta=\mathrm{i} \omega ; \omega=(2 n \pi / l) \in \mathbb{R}$. This implies

$$
\left(\begin{array}{cc}
k_{, \zeta_{1}} v_{1}-\Lambda & k_{, \zeta_{2}} v_{1}-k \zeta \\
k_{\zeta_{1}} v_{2}+k \zeta & k_{\zeta_{2}} v_{2}-\Lambda
\end{array}\right)\left(\begin{array}{c}
\hat{\theta}_{1} e^{\zeta s} \\
\hat{\theta}_{2} e^{\zeta s}
\end{array}\right)=\left(\begin{array}{l}
0 \\
0
\end{array}\right)
$$

and finally

$$
p(\Lambda)=\Lambda^{2}-\frac{\partial k}{\partial \rho} \Lambda+k \frac{\partial k}{\partial s} \Lambda+k^{2} \zeta^{2}=0 .
$$

If $k$ is constant (cylindrical arch for instance), the polynomial $p$ becomes $p(\Lambda)=\Lambda^{2}+k^{2} \zeta^{2}=\Lambda^{2}-k^{2} \omega^{2}$ and the eigenvalues of $D^{(2)}$ are

$$
\lambda= \pm \frac{G_{\mathrm{c}}}{2} k\left(\frac{2 \pi n}{l}\right), \quad n \in \mathbb{N} .
$$

We conclude that the spectrum is symmetrical with respect to zero.

\section{References}

[1] S.P. Joshi, C.T. Sun, Impact induced fracture in a laminated composite, J. Compos. Mater. 19 (1985) 51-66.

[2] Y. Ousset, Numerical simulation of delamination growth in layered composite plates, Eur. J. Mech. A/Solids 18 (1999) $291-312$.

[3] Y. Ousset, A. Münch, Delamination growth simulation in layered composite panels, Eur. Conf. Comput. Mech., June 26-29, 2001.

[4] P. Ladeveze, A damage computational method for composite structures, Comput. Struct. 22 (1992) $79-87$. 
[5] O. Allix, A. Corigliano, Geometrical and interfacial non-linearities in the analysis of delamination in composites, Int. J. Solids Struct. 36 (1999) 2189-2216.

[6] J.L. Chaboche, F. Feyel, Y. Monerie, Interface debonding models: a viscous regularization with a limited rate dependency, Int. J. Solids Struct. 38 (2001) 3127-3160.

[7] G. Alfano, M.A. Crisfield, Finite element interface models for the delamination analysis of laminated composites: mechanical and computational issues, Int. J. Numer. Meth. Engrg. 50 (2001) 1701-1736.

[8] V. Lazarus, Fatigue propagation path of 3D plane crack under mode I loading, C.R. Acad. Sci. Paris, Série II b 327 (1999) $1319-$ 1324.

[9] J.B. Leblond, V. Lazarus, S. Mouchrif, Crack paths in three-dimensional elastic solids. II. Three-term expansion of the stress intensity factors - applications and perspectives, Int J. Solids Struct. 36 (1999) 105-142.

[10] X. Li, M. Keer, A direct method for solving crack growth problems-I, Int. J. Solids Struct. 29 (1992) $2735-2747$.

[11] E.F. Rybicki, A. Kanninen, A finite element calculation of stress intensity factors by a modified crack closure integral, Engrg. Fract. Mech. 9 (1977) 931-938.

[12] B. Cochelin, M. Pottier-Ferry, A numerical method for buckling and growth of delamination in composite laminates, Comput. Meth. Appl. Mech. Engrg. 89 (1991) 443-459.

[13] B. Storakers, B. Andersson, Nonlinear plate theory applied to delamination in composites, J. Mech. Phys. Solids 36 (1988) 689-718.

[14] P. Destuynder, M. Djaoua, Sur une interprétation mathématique de 1'intégrale de Rice en théorie de la rupture fragile, Math. Meth. Appl. Sci. 3 (1981) 179-207.

[15] M. Bonnet, Stability of crack fronts under Griffith criterion: a computational approach using integral equations and domain derivatives of potential energy, Comp. Meth. Appl. Mech. Engrg. 173 (1999) 337-364.

[16] A.A. Griffith, The phenomena of rupture and flows in solids, Philos. Trans. Royal Soc. London, Series A 221 (1921) 163198.

[17] A. Friedman, Y. Liu, Propagation of cracks in elastic media, Arch. Rational Mech. Anal. (1996) 235-290.

[18] S.A. Nazarov, O.R. Polyakova, Rupture criteria, asymptotic conditions at crack tips and selfad-joint extensions of the Lamé operator, Trans. Moscow Math. Soc. 57 (1996) 13-66.

[19] R.M. Pradeilles-Duval, C. Stolz, Sur le problème d'évolution des solides avec changement de phase irréversible, C.R. Acad. Sci. Paris, Série II b 313 (1991) 297-302.

[20] G.A. Francfort, J.J. Marigo, Revisiting brittle fracture as an energy minimisation problem, J. Mech. Phys. Solids 46 (8) (1998) 1319-1342.

[21] N. Point, E. Sacco, A delamination model for laminated composites, Int. J. Solids Struct. 33 (1996) $483-509$.

[22] M.A. Tzaferopoulos, P.D. Panagiotopoulos, Delamination of composites as a substationarity problem: numerical approximation and algorithms, Comput. Meth. Appl. Mech. Engrg. 110 (1993) 63-85.

[23] Z. Cheng, S. Kitipornchai, Nonlinear theory for composite laminated shells with interfacial damage, J. Appl. Mech. 65 (1998) 711-718.

[24] A. Münch, Y. Ousset, Taux de restitution de l'énergie pour une poutre courbe mince, C.R. Acad. Sci. Paris, Série II b 328 (2000) $471-476$.

[25] F. Ozdil, L.A. Carlsson, Characterization of mode I delamination growth in glasse/epoxy composite cylinders, J. Compos. Mater. 34 (5) (2000) 420-441.

[26] P. Davies, B. Gineste, F. Pierron, L.A. Carlsson, Delamination resistance of cylindrical composite structures, XII ${ }^{\text {ieme }}$ Journées Nationales sur les Composites, ENS Cachan, 2000.

[27] B. Storakers, P.L. Larsson, On buckling and growth of shallow delaminations, in: A. Benallal (Ed.), Continuous Damage and Fracture, 2000, pp. 187-195.

[28] C.C. Rankin, F.A. Brogan, E. Riks, Some computational tools for the analysis of through cracks in stiffened fuselage shells, J. Comput. Mech. 13 (1993) 143-156.

[29] M. Charlotte, G. Francfort, J.J. Marigo, T. Truskinovski, Revisiting brittle fracture as an energy minimization problem: comparisons of Griffith and Barenblatt surface energy models, in: A. Benallal (Ed.), Continuous Damage and Fracture, 2000, pp. $7-18$.

[30] P. Ciarlet, Mathematical Elasticity Vol. III: Theory of Shells, Studies in Mathematics and its Application, North-Holland, Amsterdam, 2000.

[31] P. Destuynder, T. Nevers, Un modèle de calcul des forces de délaminage dans les plaques minces multicouches, J. Mec. Théor. Appl. 6 (1987) 179-207.

[32] Q.S. Nguyen, Stability and Nonlinear Solids Mechanics, Wiley, Chichester, 2000.

[33] N. Möes, J. Dolbow, T. Belytschko, A finite element method for crack growth without remeshing, Int. J. Numer. Meth. Engrg. 46 (1) (1999) 131-150.

[34] G.N. Wells, L.J. Sluys, A new method for modelling cohesive cracks using finite elements, Int. J. Numer. Meth. Engrg. 50 (2001) $2667-2682$. 
[35] M. Bernadou, Méthodes d'Éléments Finis Pour les Problèmes de Coques Minces, Recherches en Mathématiques Appliquées, Masson, Paris, 1994.

[36] B.D. Davidson, An analytical investigation of delamination front curvature in double cantilever beam specimens, J. Compos. Mater. 24 (1990) 1124-1137. 